PALEO

Revue d'archéologie préhistorique

$27 \mid 2016$

Varia

\title{
Le Châtelperronien de Germolles (Grotte de La Verpillière I, commune de Mellecey, Saône-et- Loire, France)
}

The Châtelperronian of Germolles (Grotte de la Verpillière I, Mellecey, Saône-etLoire, France)

Harald Floss, Christian Hoyer et Heike Würschem

\section{OpenEdition \\ Journals}

Édition électronique

URL : http://journals.openedition.org/paleo/3208

DOI : $10.4000 /$ paleo.3208

ISSN : 2101-0420

Éditeur

SAMRA

Édition imprimée

Date de publication : 30 décembre 2016

Pagination : 149-176

ISSN : 1145-3370

Référence électronique

Harald Floss, Christian Hoyer et Heike Würschem, « Le Châtelperronien de Germolles (Grotte de La Verpillière I, commune de Mellecey, Saône-et-Loire, France) », PALEO [En ligne], 27 | 2016, mis en ligne le 01 juin 2018, consulté le 10 décembre 2020. URL : http://journals.openedition.org/paleo/3208 DOI : https://doi.org/10.4000/paleo.3208

\section{(c) $)(9)$}

PALEO est mis à disposition selon les termes de la licence Creative Commons Attribution - Pas d'Utilisation Commerciale - Pas de Modification 4.0 International. 


\title{
Le Châtelperronien de Germolles (Grotte de La Verpillière I,
} commune de Mellecey, Saône-et-Loire, France)

\author{
Harald FLOSS ${ }^{(a, b)}$, Christian HOYER ${ }^{(a, b)}$, Heike WÜRSCHEM ${ }^{(a)}$
}

\begin{abstract}
Résumé : La grotte de La Verpillière Verpillière à Germolles, commune de Mellecey (Saône-et-Loire), a produit une séquence archéologique allant du Paléolithique moyen jusqu'au Gravettien. Cette contribution se focalise sur l'industrie lithique châtelperronienne, en prenant en considération 150 ans de recherches dans la cavité. D'un point de vue technoet typologique, cette étude tend à inclure la totalité des pièces de ce site appartenant probablement au complexe châtelperronien. Elle comprend également les observations de nos propres fouilles, incluant un niveau châtelperronien en place. La série lithique de Germolles est placée dans le contexte du Châtelperronien en Bourgogne méridionale et dans l'Est de la France.
\end{abstract}

Mots-clés : Châtelperronien, Germolles, Grotte de La Verpillère I, analyses techno-typologiques, Bourgogne méridionale, Est de la France.

Abstract: The Châtelperronian of Germolles (Grotte de la Verpillière I, Mellecey, Saône-et-Loire, France). The Grotte de la Verpillière I in Germolles (Saône-et-Loire, France) has yielded an archaeological sequence from the Middle Palaeolithic to the Gravettian period. This contribution focusses on the Châtelperronian lithic industry, covering 150 years of research. From a techno-typological point of view, all known lithic objects presumably dating to this period are subject of this investigation. This study includes in-situ observations of Châtelperronian layers, as part of our own recent excavations. The lithic assemblage is put into context with other Châtelperronian samples in Southern Burgundy and Eastern France.

Key-words: Châtelperronian, Germolles, Grotte de La Verpillière I, techno-typological analysis, Southern Burgundy, Eastern France.

\section{Introduction}

Depuis 2006, une équipe de l'université de Tübingen, sous la direction d'Harald Floss, travaille aux grottes de Germolles, dites aussi " grottes de La Verpillière ", situées sur la commune de Mellecey, à quelques kilomètres à l'ouest de Chalon-sur-Saône (fig. 1). Elles sont orientées au nord, sur un flanc de la vallée de l'Orbize, petit affluent de la Saône. La Côte chalonnaise a été fréquentée dès le Paléolithique moyen et héberge plusieurs sites en grotte et en plein air, dont Saint-Martin-sous-Montaigu, Saint-Denisde-Vaux et les grottes de Rully (Gros et Gros 2005). C'est pourquoi nous avons mis en place en 2014, avec Yves Pautrat du S.R.A. Bourgogne (Dijon), un PCR consacré au Paléolithique de la Bourgogne méridionale, qui fédère aujourd'hui une cinquantaine de chercheurs (Floss et al. $2015,2016)$. Les recherches et travaux de terrain au sein de la grotte de Germolles ont commencé au cours des années 1860. L'historique des fouilles n'est pas simple. Ici nous souhaitons et pensons avoir l'obligation de décortiquer les données essentielles trop souvent noyées dans un lacis d'informations plus ou moins précises. Pour le Châtelperronien, une des occupations les plus emblématiques de cette grotte, ce constat se décline à travers quelques témoignages écrits, pour n'en citer que quelques-uns : Nelly Connet (2002 - p. 9) : « La grotte de Germolles (Saône-et-Loire) aurait renfermé des pointes de Châtelperron et peut-être un niveau châtelperronien malheureusement indifférencié des autres niveaux d'occupation de la cavité. » ou encore François Bachellerie

(a) Eberhard Karls Universität Tübingen, Institut für Ur- und Frühgeschichte und Archäologie des Mittelalters, Abteilung Ältere Urgeschichte und Quartärökologie, Schloss, Burgsteige 11, DE-72070 Tübingen - harald.floss@uni-tuebingen.de ; christian.hoyer@uni-tuebingen.de; heike.wuerschem@student.uni-tuebingen.de

(b) UMR6298 ARTeHIS, PCR «Le Paléolithique supérieur en Bourgogne méridionale », Université de Bourgogne, 6 Boulevard Gabriel, FR-21000 Dijon - 


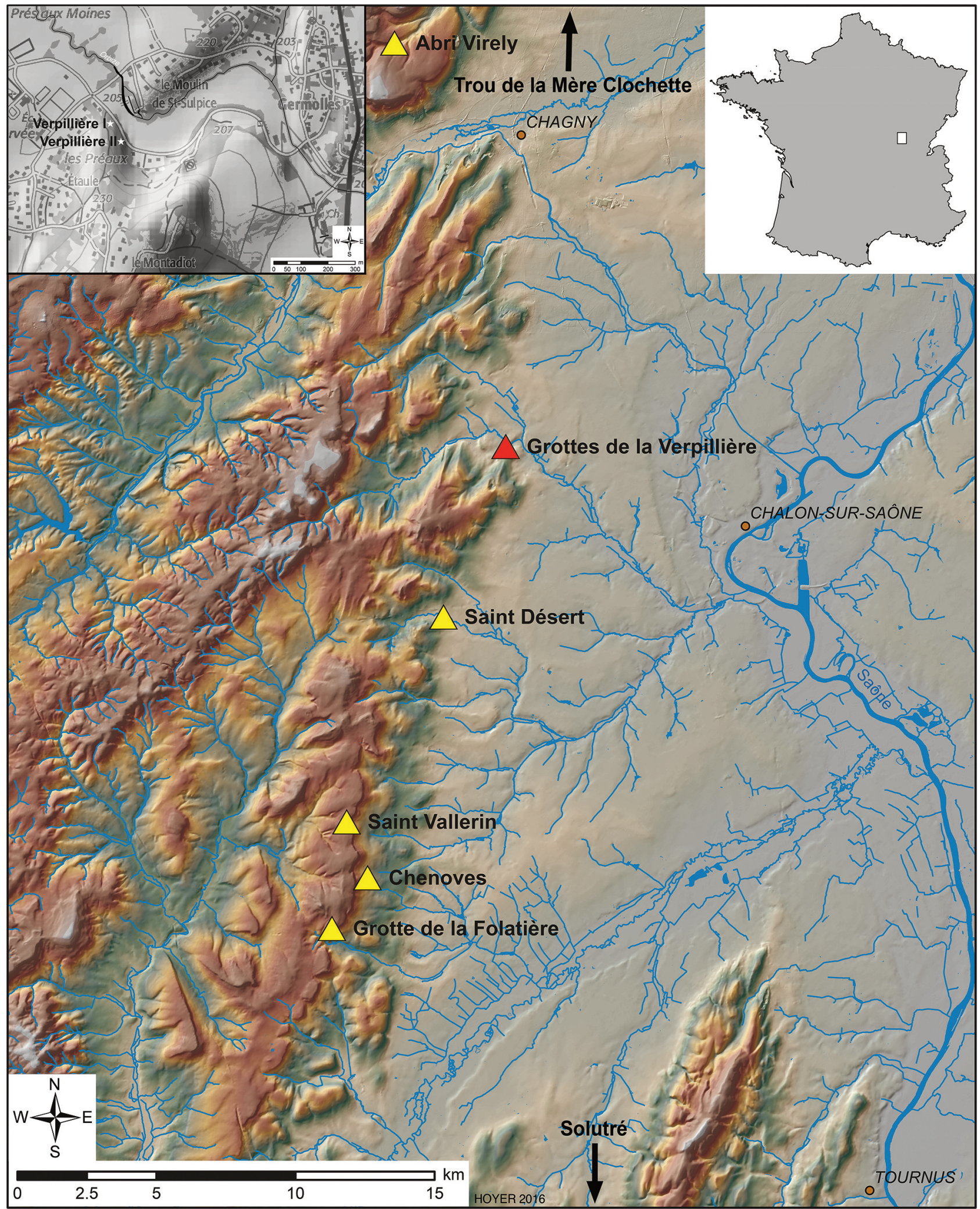

Figure 1 - Situation de la Grotte de La Verpillière I à Germolles et des sites châtelperroniens en Bourgogne méridionale (données de base : IGN France ; cartographie : Ch. Hoyer).

Figure 1 - Location of the Grotte de la Verpillière I (Germolles) and other châtelperronian sites in Southern Burgundy (base map : IGN France ; mapping : Ch. Hoyer). 
(2011 - p. 321) au regard de Germolles : « fouilles anciennes, pas d'informations stratigraphiques claires, peu de matériel » (...).

Le but de notre contribution est donc de rassembler, le plus objectivement possible, les données existantes sur le Châtelperronien de Germolles, aussi bien pour les fouilles anciennes que pour nos propres travaux. Ce projet connaît des limites, mais aussi un potentiel non négligeable. En l'état actuel de nos connaissances, Germolles constitue le site le plus oriental du Châtelperronien, à $115 \mathrm{~km}$ de distance d'Arcy-sur-Cure, $225 \mathrm{~km}$ d'Ormesson et $300 \mathrm{~km}$ des Cottés. Probablement porté par les derniers Néandertaliens et proche des régions contemporaines d'ores et déjà occupées par Homo sapiens (Aurignacien du Jura souabe) (Floss 2003), Germolles occupe une situation paléogéographique extraordinaire et mérite une étude, malgré les insuffisances de la conservation archéologique.

\section{La chronologie de l'occupation paléolithique de la Grotte de La Verpillière I à Germolles}

Après 150 ans de fouilles et d'explorations, il convient, avant se pencher sur la question du Châtelperronien, de dresser un tableau succinct des phases d'occupation de cette grotte, en reliant les résultats des fouilles anciennes (1868 - 1959, fig. 2) à ceux de nos propres investigations à partir de 2006. Pour les fouilles récentes effectuées par nos soins, nous nous référons à un système de dénomination stratigraphique suivant les unités géologiques (GH's) rencontrées, parfois identiques aux niveaux archéologiques, parfois en contenant plusieurs. La numérotation ne suit pas l'attribution chronologique des niveaux mais l'ordre successif de leurs découvertes, ainsi que des homogénéisations post-fouilles.

L'occupation de la grotte de La Verpillière I commence au Paléolithique moyen, elle est marquée par le niveau GH 16, qui est en place sur plusieurs mètres carrés à l'ouest de l'intérieur de la grotte. II permet des observations palethnologiques de la structuration interne de l'occupation néandertalienne (Floss et al. 2010 ; Litzenberg 2015 ; Hoyer et al. 2016). Ce niveau contient, d'un point de vue archéologique, un Moustérien classique avec présence de débitage Levallois, et aussi de quelques éléments bifaciaux. Nous disposons d'une datation ESR /U-Th de $51000 \pm 3000$ à $48000 \pm 3000$ pour une dent d'herbivore, se situant vers la transition entre ce GH16 et le GH15 (Richard et al. 2016a; Richard et al. 2016b). Nous pouvons donc en déduire que le niveau $\mathrm{GH} 16$ présente une datation un peu plus ancienne que $50000 \mathrm{BP}$, dans une phase froide du début de l'OIS 3 . Malgré la présence d'éléments bifaciaux, ce niveau se distingue archéologiquement du faciès Paléolithique moyen le plus emblématique pour le site qui est le Micoquien, au sens large, dénommé aussi groupes à Keilmesser, caractérisé par la présence des bifaces à dos aménagés, d'un ou plusieurs coups de tranchet oblique(Pradniks) et popularisé, sur la base des séries anciennes, par René Desbrosse $(\dagger)$ (Desbrosse et Texier 1973). Contrairement à la nouvelle grotte de La
Verpillière II où nous disposons d'un niveau stratifié de ce complexe, nos fouilles récentes à La Verpillière I n'ont jamais pu trouver ce type d'industrie dans un niveau intact. Ce n'est qu'en 2015 que nous avons découvert, sur la terrasse de cette grotte, quelques indices de la position stratigraphique de ce type de Paléolithique moyen (GH44 à $\mathrm{GH} 45)$. En ce qui concerne les datations de ces groupes à Keilmesser à Germolles, nous nous référons à la grotte voisine de La Verpillière II où ce type d'industrie (Frick \& Floss 2017) a récemment obtenu des datations minimales par thermoluminescence de 47500 BP pour le niveau inférieur $\mathrm{GH} 4$, de $45000 \mathrm{BP}$ pour le niveau supérieur $\mathrm{GH} 3$ (Zöller \& Schmidt 2016) et une datation ${ }^{14} \mathrm{C}$ de $>48200 \mathrm{BP}$ (Heckel et al. 2016).

Au sein des séries lithiques provenant des fouilles anciennes, il a été remarqué la présence de petits bifaces symétriques, en partie de forme triangulaire à cordiforme, s'intégrant pleinement d'un point de vue technotypologique, à un Moustérien de tradition acheuléenne (voir Soressi 2002). Pour ce type de Paléolithique moyen, nous en sommes au même point que pour les groupes à Keilmesser : à La Verpillière I, nous n'avons jamais trouvé une telle industrie dans un niveau en place. Nous tenons à rappeler la présence, dans les séries anciennes, de quelques très grands bifaces qui ont suggéré l'existence d'un véritable Acheuléen (Gros et Gros 2005). Mais nous n'avons, dans l'état actuel de nos recherches, aucun indice de la présence de ce complexe emblématique du Paléolithique inférieur. D'ailleurs, dans le nord de la Bourgogne, des bifaces de forme régulière ont été signalés dans le OIS 5 (Deloze et al. 1994).

Dans l'ordre chronologique, suit le Châtelperronien, le motif essentiel de cette contribution. Heureusement, la fouille de 2014 a permis, dans le centre de la grotte, de dénicher ce complexe dans un niveau intact (GH40), totalement coloré en rouge par la présence d'oxydes de fer (hématite) (fig. 3). Nous avons obtenu, pour ce niveau, une datation ${ }^{14} \mathrm{C}$ sur os de $49600 \pm 3900$ cal. BP (OxA 32 235). Cette datation nous paraît un peu trop ancienne, notamment en présence des datations châtelperroniennes actuelles pour Arcy-surCure (Grotte du Renne) qui varient entre 40500 et 45000 cal. BP (Soressi et Roussel 2014 ; Hublin et al. 2012).

En ce qui concerne la stratigraphie dans ce secteur (fig. 3), c'est la seule zone dans La Verpillière I où a été trouvé le Châtelperronien en place. La coupe débute à la base sur la roche mère $(\mathrm{GH} 6)$, en passant par les niveaux stériles (GH 2, 4, 22), le Moustérien du GH 15b (qui correspond au GH16 dans l'ouest de la grotte), les GHs 41 (Moustérien), le Châtelperronien (GH 40) jusqu'à l'Aurignacien (GH 15c, tombé vers le bas, suite aux fouilles anciennes).

En ce qui concerne l'Aurignacien, nous avons pu récemment détecter la présence du Protoaurignacien à Germolles, au vu de certains types de nucléus à débitage croisé et de lamelles Dufour, provenant des fouilles anciennes (Wegeng 2015 ; Wegeng \& Floss 2016). Sans indice stratigraphique, cette déclaration, issue d'observations purement technologiques, ne tient pas 


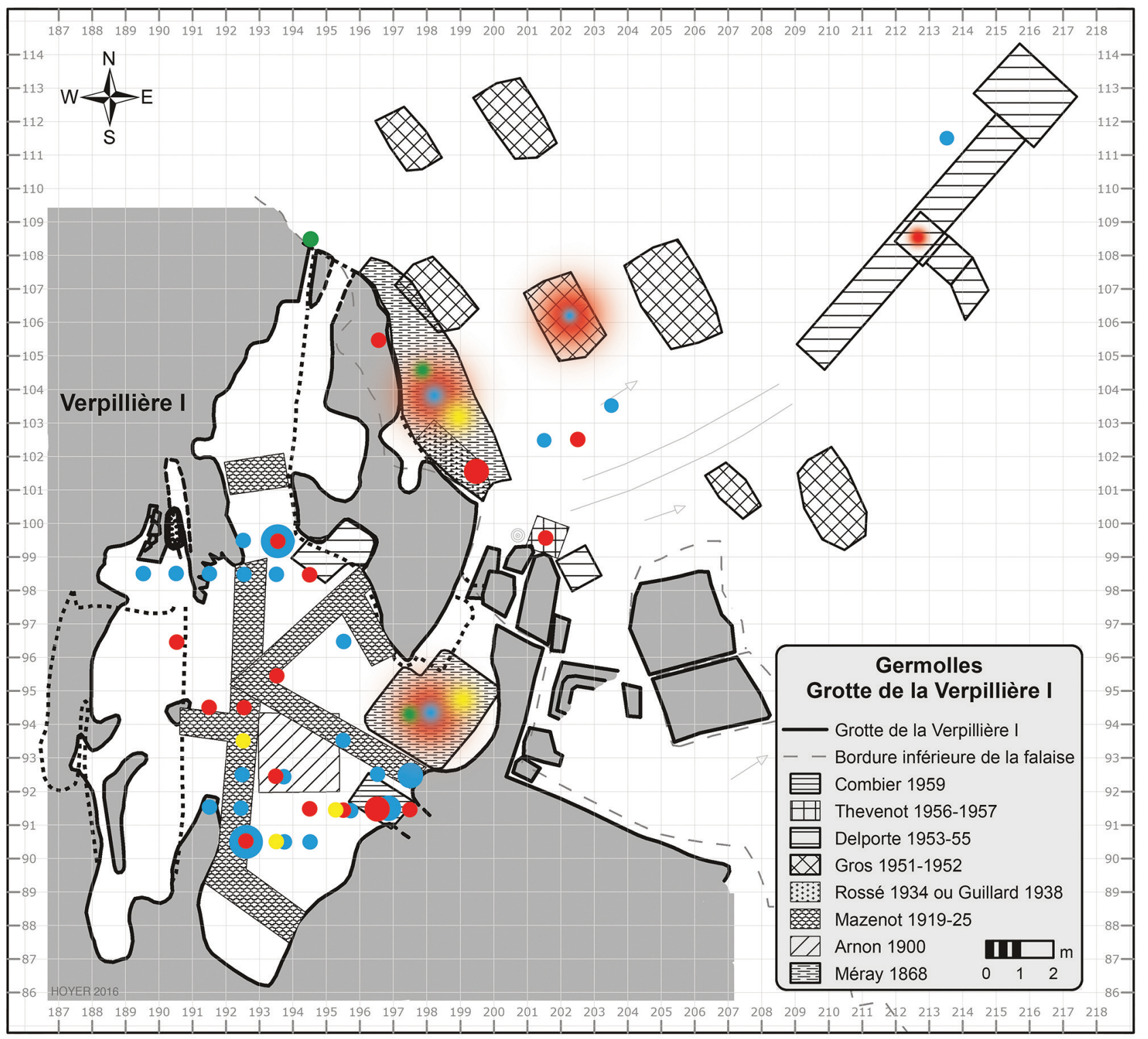

Figure 2 - Plan de la Grotte de La Verpillière I avec quadrillage des fouilles récentes (Université de Tübingen) et signatures des diverses fouilles anciennes. Répartition des vestiges Châtelperroniens : en rouge : pointes de Châtelperron, en bleu : débitage (lames), en jaune : nucléus, en vert : couteaux. Le diamètre des symboles reflète le nombre de pièces de 1 à 8 (réalisation: Ch. Hoyer).

Figure 2 - Site plan of the Grotte de la Verpillière I. Charted are the squares recently excavated by the team of the University of Tübingen and the areas of ancient excavations. Distribution of the châtelperronian artefacts : red : châtelperronian points, blue : blanks (blades), yellow : cores, green : couteaux. The diameter of the symbols reflects the number of pieces, ranging from 1 to 8 (realization: Ch. Hoyer).

compte de la discussion actuelle sur la genèse (ou devrions-nous dire l'existence ?) du Protoaurignacien, soit comme phase initiale de l'Aurignacien, soit comme phénomène technologique à l'intérieur du corpus de l'Aurignacien, sans valeur chronologique.

L'Aurignacien ancien est connu depuis les fouilles anciennes et il a fourni des industries lithiques et osseuses spectaculaires et très riches. Notons par exemple le très grand nombre de pièces carénées épaisses et classiques (voir Floss 2005 ; Floss et al. 2013 b, 2015 a), qui indiquent un débitage lamellaire intensif sur place, ainsi que l'industrie en os, en bois et en ivoire également particulièrement riche et montrant des indices d'une production sur place (Tartar \& Heckel 2016 ; Floss et al. 2015 a). Nous n'avons jamais retrouvé ce même type d'Aurignacien (carénés épais, sagaies à base fendue, etc.) dans des niveaux intacts, au cours de nos propres fouilles. Cela ne signifie pas que nous n'ayons pas rencontré de faciès aurignacien, en tout cas pas dans une spécificité 

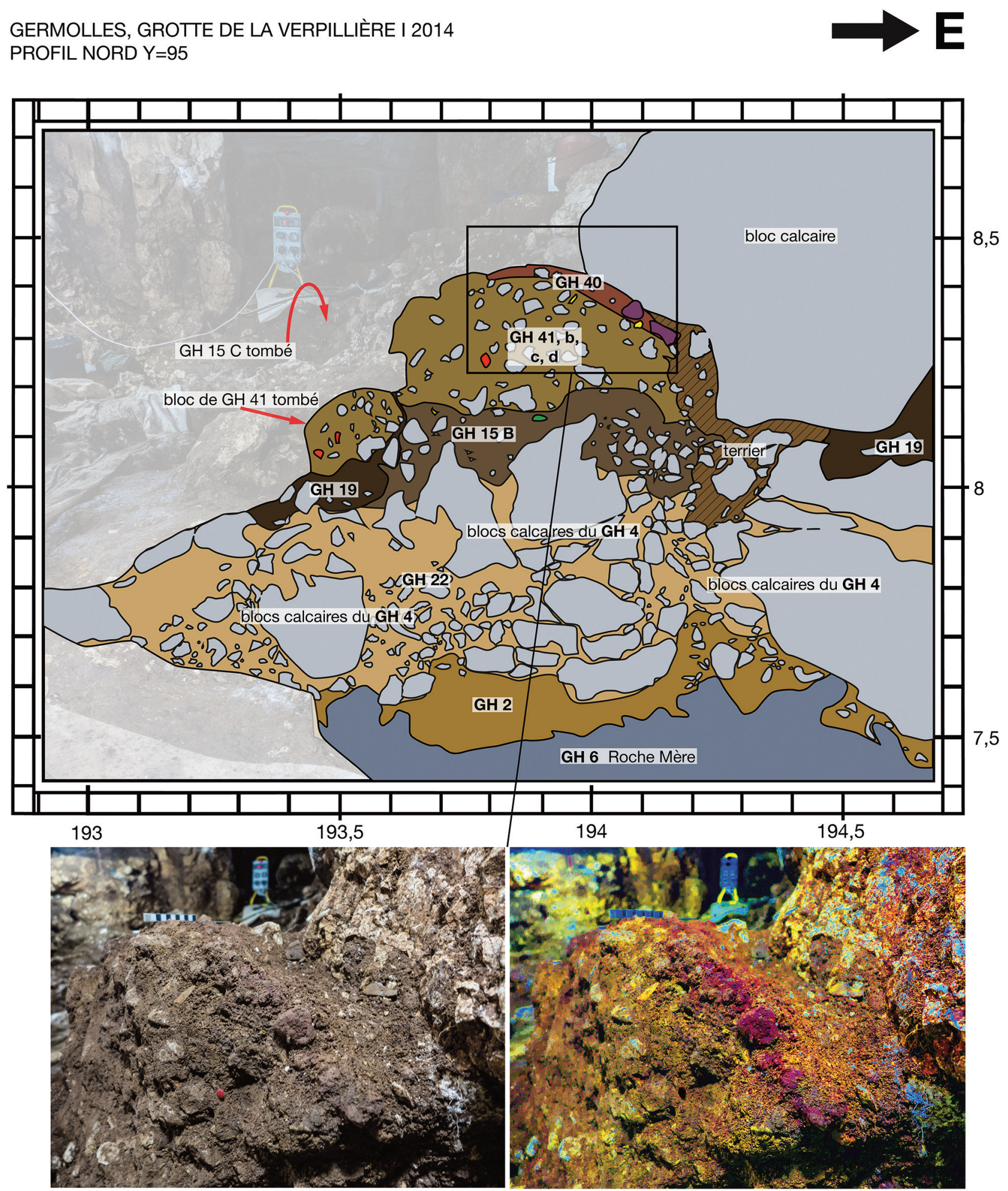

Figure 3 - Grotte de La Verpillière I, le Châtelperronien du niveau GH4O dans son contexte stratigraphique. En haut : coupe stratigraphique sur la base d'une prise photogrammétrique. En bas, à gauche : prise photographique du niveau GH 40, en bas à droite prise photographique du niveau GH 40, modifiée au moyen du logiciel DStretch (photos et réalisation $\mathrm{CH}$. Hoyer).

Figure 3 - Grotte de la Verpillière I, the Châtelperronian layer GH 40 in its stratigraphical context. On top : profile image based on photogrammetry. Bottom left : Photography of the layer GH 40. Bottom right : Photography of the Layer GH 40, modified by DStretch software (Photos and realization : Ch. Hoyer). 
aussi classique que celle des séries anciennes. Nous avons rencontré l'Aurignacien, ou au moins un Paléolithique supérieur ancien d'affinités aurignaciennes, dans plusieurs niveaux et à plusieurs endroits de la cavité (fig. 3) : les niveaux les plus représentatifs de l'Aurignacien sont le GH24 et le GH35. Sur une surface de $1,5 \mathrm{~m}^{2}$ à l'ouest de la grotte, l'industrie lithique aurignacienne du gh35 se caractérise par plusieurs nucléus à lamelles et aussi des supports issus de ce type de débitage (floss et al. 2013 a, fig. 27). En ce qui concerne la datation absolue de l'Aurignacien, nous disposons d'une date $>44300 \mathrm{BP}$ sur os (OxA 32 228) pour le GH24, datation certes un peu trop ancienne, mais néanmoins pas non plus choquante. On a aussi daté, au cours de ces dernières années, de nombreux éléments de l'industrie osseuse clairement aurignacienne (sagaies, lissoirs, poinçons). Ces datations (GrA-49 115 à 49 127) tournent autour de 32000 BP. Elles sont certes assez récentes. Toutefois, l'analyse de l'industrie lamellaire issue des niveaux remaniés de La Verpillière I (Kremmer 2015) a pu mettre en évidence la présence de certains types de lamelles, retouchées ou non, évoquant la présence d'un Aurignacien évolué (Chiotti 2003 ; Pesesse et Michel 2006).

Le Gravettien n'a été reconnu avec certitude, pour la première fois, que dans le cadre de nos fouilles récentes. D'abord ont été reconnus quelques fossiles-directeurs lithiques, micro-gravettes et aussi, élément inédit dans cette région de la Bourgogne du sud, quelques burins de Noailles. Au cours de nos fouilles de 2009 (Floss et al. 2009), nous avons découvert, à l'extérieur de la grotte actuelle, à quelques mètres au nord de l'entrée, une zone riche en vestiges gravettiens, contenant aussi des éléments de parure (dents percées). Cet ensemble a été daté, par plusieurs mesures ${ }^{14} \mathrm{C}$ (GrA-44 701 à 44 704, 45 482, 45 450 ) entre $26010 \pm 120$ et $28900 \pm 440$ BP. Nous avons également rencontré un dépôt gravettien à l'intérieur de la grotte. C'est le GH23, tout en haut d'un cône d'éboulis à l'ouest de la grotte, contenant quelques éléments à dos abattu.

La succession chronologique de la grotte de La Verpillière I se termine, après 30000 ans d'occupation humaine, avec l'éventuelle présence du Solutréen, uniquement sous forme d'un ou deux fragments de feuilles de laurier, vestiges isolés et rencontrés, avec une étiquette "Germolles 》, dans la collection du laboratoire de Géologie de l'Université de Lyon I (Dutkiewicz \& Floss 2015). Nous constatons l'absence du Magdalénien et l'occupation se termine par quelques vestiges isolés postglaciaires.

\section{Le Châtelperronien à Germolles}

Depuis sa découverte en 1868, la grotte de La Verpillière à Germolles a connu un historique de la recherche long et compliqué (Dutkiewicz \& Floss 2015). Les dernières fouilles coordonnées, avant nos propres travaux, ont eu lieu au cours des années 1950, respectivement par André-Charles Gros, Jean Combier, Jean-Paul Thévenot et Henri Delporte, ce dernier s'intéressant surtout au Châtelperronien, à la suite de ses travaux entrepris sur le site éponyme dans
l'Allier (Delporte 1955). Après un demi-siècle d'abandon et d'explorations non-coordonnées, l'équipe de Tübingen s'intéresse à ce site depuis 2003 , en établissant un plan détaillé de la grotte et en y effectuant des fouilles programmées depuis 2006. Nos travaux ont permis, en 2006, sous le prolongement de la même corniche, la découverte d'une nouvelle grotte. Désormais, la grotte du $19^{e}$ siècle porte le nom de Verpillière I et la nouvelle grotte celle de Verpillière II.

En ce qui concerne le Châtelperronien, ce n'est que la grotte I qui a livré des données ; à l'exception de quelques éléments peu importants provenant de la grotte II. Et ce n'est pas un moindre fait que le découvreur du site, Charles Méray, décrit et figure, à partir de 1869 , les pièces ultérieurement reconnues comme fossile-directeurs de ce complexe, les pointes de Châtelperron, en les appelant « couteaux » (Méray 1869) ou " couteau présentant un dos taillé avec soin » (Méray et Chabas 1876, fig. 13). Charles Méray décrit également une autre pièce (1876, fig. 14), aujourd'hui appartenant au corpus nous intéressant, comme « perçoir (...), terminé en pointe recourbée et taillée» (fig. 6.1).

Au début du $20^{\circ}$ siècle, le site de Germolles est mentionné dans la fameuse bataille aurignacienne. Henri Breuil (1909 - p. 14), sans y entreprendre de propres fouilles, incorpore le site dans son argumentation sur la position chronologique de l'Aurignacien au début du Paléolithique supérieur. En se basant sur les découvertes de Ch. Méray, ce même auteur (Breuil 1911 - p. 39) décrit « plusieurs lames à retouche unilatérale, soit localisée vers l'extrémité pointue, soit intéressant tout un côté entièrement abattu » et les considère "sans aucun doute possible, du type de Châtelperron ". Néanmoins, il déplore le manque de contexte de ces pièces, qui sont en plus assez rares, et en déduit un niveau peu important du "Périgordien ancien ». Malgré le renom gagné et la découverte de quelques pièces châtelperroniennes issues des fouilles de la première moitié du $20^{\circ}$ siècle (Dutkiewicz 2011 ; Dutkiewicz \& Floss 2015), ce n'est qu'à partir des années 1950 que les recherches à Germolles et l'intérêt du Châtelperronien regagnent de l'importance. Accompagné par les travaux d'Armand-Calliat (1950), de l'Abbé Guillard (1954) (Dutkiewicz 2011, fig. 40) ainsi que ceux du jeune AndréCharles Gros (1958), c'est notamment une situation de compétition entre Jean Combier et Henri Delporte autour de ce site qui fait avancer les choses. Aujourd'hui c'est beaucoup plus la lecture d'une correspondance inédite entre ces deux auteurs que leurs propres publications (Delporte 1953, 1955 ; Combier 1955, 1959) qui fait comprendre la discussion. Les différences entre ces deux chercheurs, relatives à leurs visions du monde archéologique, étaient importantes. Se basant sur les fouilles anciennes et sur leurs propres travaux (sondage Jean Combier 1959 à l'intérieur de la grotte, fouilles et notamment grande tranchée d'Henri Delporte devant l'entrée de la grotte entre 1953 et 1955), les points principaux de la discussion tournent autour de la question de l'importance de l'Aurignacien (moindre pour Delporte et pour Combier, à juste titre, importante) et de l'existence d'un 
Châtelperronien indépendant. Cette dernière idée soutenue par Henri Delporte et appelée "Castelperronien pur » (Delporte 1953 - p. 238) ou « Périgordien inférieur (Delporte 1955 - p. 160) est contestée par Jean Combier (1952, inédit) qui considérait, au début de la discussion, les quelques pointes de Châtelperron comme un élément du Moustérien tardif ou bien d'une composante de l'Aurignacien. Plus tard, les deux chercheurs arrivèrent à s'accorder, dans un communiqué commun (inédit, sans année) d'après lequel, malgré le manque de résultats stratigraphiques, le Châtelperronien précédait un Aurignacien, quant à lui différencié en plusieurs niveaux (voir aussi Combier 1955 p. 603). Jean Combier (1959 - p. 121) décomptait alors une vingtaine de pointes de Châtelperron, issues de plusieurs collections, mais son point de vue critique concernant la présence du Châtelperronien perdura (Combier $1989-\mathrm{p}$. 274, 1990 - fig. 1). Selon lui, tout en ignorant totalement le caractère gravement remanié des séries étudiées, Germolles ne faisait pas partie de l'aire de répartition du Châtelperronien, mais, comme le Trou de la Mère Clochette, se trouvait en relation, certes non-définie, avec ce dernier : "À Germolles, près de Chalon-sur-Saône, grotte au porche effondré, qui figure à la suite des observations d'H. Breuil en 1911, dans la liste des sites du "vieil Aurignacien » (devenu pro parte le Châtelperronien), on n'observe que quelques rares couteaux à dos typiques noyés dans un outillage aurignacien très abondant et bien caractérisé par ses pointes en os... » (Combier 1990 - p. 270, voir aussi 277).

En ce qui concerne les fouilles importantes d'Henri Delporte (grande tranchée à l'extérieur et plusieurs sondages à l'intérieur et à l'extérieur de la grotte) (1955 - p. 160), les résultats stratigraphiques se sont avérés décevants, seuls quelques éléments châtelperroniens dispersés ayant été découverts dans l'éboulis du niveau 1. Jean Combier, quant à lui, effectue, en 1959, un petit sondage à l'intérieur de la grotte et constitue une stratigraphie qui a été mise en valeur par nos soins (fig. 4) (Floss et al. 2013 b). Le niveau 4 de ce petit témoin stratigraphique d'une épaisseur de $10 \mathrm{~cm}$, a été appelé " Moustérien à éclats ou Châtelperronien " et constituait, avec une certaine bienveillance, le premier témoin d'un Châtelperronien de ce site en place (voir aussi Floss et al. 2013 b). Aujourd'hui, après étude de la série (Dutkiewicz \& Floss 2015), nous voyons ces résultats de manière plus sceptique car parmi les quelques 19 pièces du niveau 4 en question, aucun élément clairement châtelperronien n'est attesté.

Une reprise des travaux sur Germolles est marquée, au cours des années 1970, par les études de René Desbrosse (Desbrosse \& Texier 1973 ; Desbrosse et al. 1976 ; Desbrosse 1982), qui confirme la présence du Châtelperronien sur place et le contextualise dans un ensemble de sites périgordiens dans l'Est de la France. Dans le cadre d'un article clé à cet égard, Desbrosse (1982) plaide en faveur d'une occupation pleinement châtelperronienne indépendante dans ce site. II mentionne aussi, à côté d'une série de huit pointes de Châtelperron (Desbrosse 1982, fig. 3), pour la première fois bien dessinées ainsi que la présence d'une pointe des Cottés
(Desbrosse 1982, fig. 3.3) et un couteau de type Abri Audi (Desbrosse 1982, fig. 3.3 et fig. 3.8).

À la suite de ces derniers travaux, l'intérêt pour le site de Germolles et son occupation châtelperronienne s'est estompé probablement parce que la grotte fut considérée comme épuisée. Au cours de ces dernières décennies, le site a été mentionné et/ou cartographié dans quelques travaux supra régionaux s'intéressant au Châtelperronien (Harrold 1986 ; Bosinski 1987, fig. 4 ; Baffier 1999 ; Roussel 2011, fig. 1.1; Roussel et Soressi 2014 - p. 34 ; Soressi et Roussel 2014, fig. 3), à Arcy-sur-Cure (Connet 2002 - p. 9), à la Côte Chalonnaise (Gros et Gros 2005, fig. 20), aux fouilles d'Henri Delporte (Gros 2007) ou à la technologie lithique châtelperronienne (Pelegrin 1995 - p. 44) témoignant l'existence du Châtelperronien à Germolles, mais sans pouvoir en dire beaucoup plus.

Le regain des travaux de la part de l'université de Tübingen à partir de 2003 a également entraîné, en plus des résultats inhérents aux propres fouilles, une nouvelle confrontation avec les données des anciennes fouilles. Plusieurs travaux ont tenté de retracer une paléogéographie dans l'Est de la France dans la zone thématique disputée par les derniers Néandertaliens et les premiers Hommes modernes en Europe (Floss 2003, 2005). Un mémoire de master (Dutkiewicz 2011 ; Dutkiewicz \& Floss 2015) s'est intéressé à une reconstitution détaillée de l'historique de la recherche de cette grotte. De plus, les nouvelles fouilles ont livré des éléments châtelperroniens, pour la plupart en contexte remanié $(\mathrm{GH} 1)$, mais également dans les niveaux de surface GH 1 et 2 de la nouvelle grotte de La Verpillière II (Götz 2013 ; Floss et al. 2013 b, fig. 3). En 2015, un premier travail universitaire s'est intéressé particulièrement à la question du Châtelperronien à Germolles (Würschem 2015).

\section{Le Châtelperronien stratifié}

Dans le cadre de nos travaux " criminologiques " de reconstitution des faits, Jean Combier nous a confié un lot de documents inédits sur Germolles. Parmi ceux-ci, se trouve un relevé stratigraphique manuscrit issu de son sondage de 1959 dans la cavité (fig. 4). Nous avons repris ce document précieux (Floss et al. 2013 b, fig. 4, voir aussi Floss et al. 2015 b - p.47 ; Dutkiewicz \& Floss 2015 - p. 27). Sur cette coupe stratigraphique, on remarque pour le niveau 4 la dénomination "Niveau Moustérien à éclats ou Châtelperronien ", ce qui a nourri l'espoir de la première indication d'un Châtelperronien en place dans cette grotte (Floss et al. 2013 b). Certes, la situation stratigraphique de ce niveau plaide en faveur d'une position vers la transition entre Paléolithique moyen et Paléolithique supérieur, représenté dans cette région juste par le Châtelperronien, mais une analyse de la série lithique de cette coupe, comptant 81 pièces au total ne révèlent pas de façon probante d'élément châtelperronien. De plus, l'intégrité du niveau 4 a été mise en question (communication orale J. Combier (voir Dutkiewicz 2011 - p. 92 ; Dutkiewicz \& Floss 2015 - p. 29). Retenons que la coupe Combier de 1959 a probablement pu mettre en évidence un niveau se 


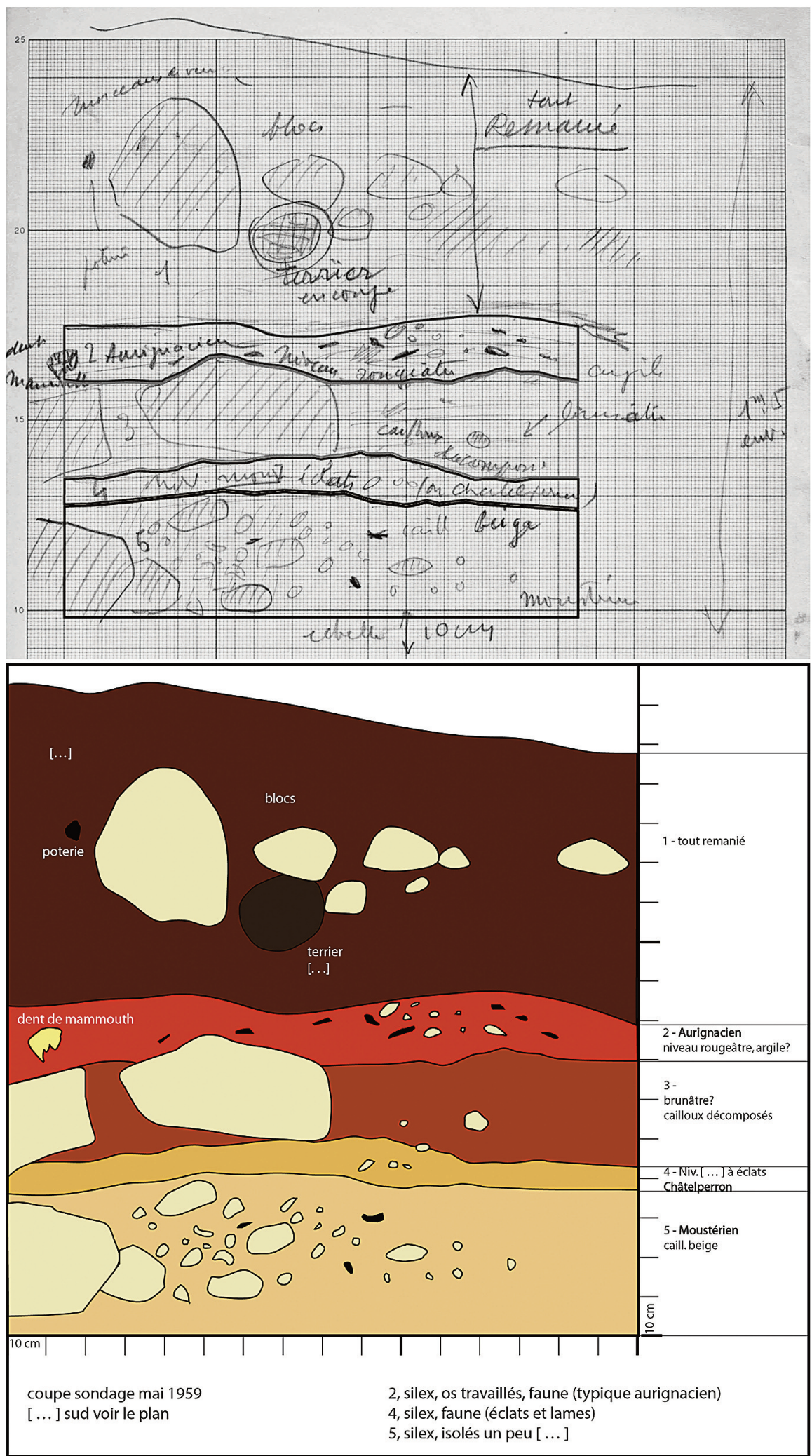

Figure 4 - Grotte de La Verpillière I, coupe stratigraphique du sondage $J$. Combier 1959, en haut : dessin d'origine par J. Combier, en bas : coupe synthétique (réalisation : E. Dutkiewicz).

Figure 4 - Grotte de la Verpillière I, stratigraphy of the sondage executed by Jean Combier in 1959, on top: sketch by J. Combier, below: synthetic stratigraphy (realization: E. Dutkiewicz). 
situant chronologiquement vers la transition entre Paléolithique moyen et supérieur, mais qu'une attribution chrono-culturelle plus poussée de la série qui y est contenue nous paraît aujourd'hui présomptueuse.

Ainsi, dès le début de nos propres travaux à La Verpillière I, nous avons pu croire à la possibilité d'une découverte d'un Châtelperronien en place (Floss et al. 2013 b - p. 336). En 2009 , nous avons pu mettre en évidence, contre une paroi proche du sondage de Jean Combier de 1959, un témoin qui a fourni huit unités stratigraphiques bien distinctes (Floss et al. 2009, fig. 37-38, voir aussi Bader 2011). Dans une position stratigraphique identique à celle mentionnée dans le sondage de J. Combier, nous avons pu découvrir, dans le niveau 12, entre autres, un fragment atypique d'une lame ou d'une pointe à dos abattu qui nous a fait appeler cette série « faciès de transition » (Floss et al. 2009 : p. 16). Plus tard, au cours des travaux dans le nord-ouest de la cavité, sous un cône d'éboulis, promettant la conservation de dépôts intacts, nous avons reconnu, dans la partie supérieure du $\mathrm{GH} 15$, un faciès portant des stigmates à la fois du Paléolithique moyen et du Paléolithique supérieur qui nous ont également fait penser à la présence d'un niveau de transition. Aujourd'hui, nous sommes convaincus que ce niveau présente une industrie mixte dûe à la présence de fortes érosions par l'eau dans cette partie de la grotte.

Ce n'est qu'en 2014 (voir Floss et al. 2014, fig. 15 ; Dutkiewicz \& Floss 2015 - p. 15 ; Floss et al. 2015 b), dans le cadre de nos fouilles au centre-est de la cavité (secteur E), que nous avons rencontré, dans les carrés de fouilles $194 / 096$ et 195/096 un niveau rapportable au Châtelperronien (fig. 3). II a été désigné, suivant la dénomination interne, GH 40. II s'agit d'un sédiment homogène sableux à limoneux avec un faible taux d'argile, observé en surface sur un tiers de mètre carré. Ce niveau se distingue par une nette coloration rouge qui doit son origine aux oxydes métalliques, non issus des processus de décomposition des calcaires voisins, mais dûe à un taux élevé de poudre d'hématite (fig. 3). Dans ce contexte, la présence d'un morceau d'hématite est à souligner. Outre une couleur rougeâtre causée par des oxydes de fer, il faut noter l'inclinaison de cette unité à $30^{\circ}$ direction du nord-est, c'est-à-dire dans l'axe d'un grand bloc calcaire (fig. 3). L'épaisseur du GH 40 ne dépasse pas les $8 \mathrm{~cm}$. Vers l'est, il continue encore un peu sous un bloc calcaire avant de disparaître. La série lithique du GH 40 est composée d'un total de 48 artefacts, dont la plupart des pièces $(n=40)$ mesurent moins d'un centimètre. La pièce la plus signifiante est une pointe de Châtelperron en silex des argiles à silex de la Côte chalonnaise (fig. 5.1). De plus, il y a une grande lame sous-crête (fig. 5.2), attribuable au même contexte chronologique, en silex local avec, sur la face dorsale, un enlèvement laminaire, des restes de préparation et du cortex. Les caractéristiques évoquent une percussion tendre, à cause de la présence d'un bulbe bien discret et d'une lèvre. II y a encore un petit fragment de lame portant des traces de feu. À côté des vestiges lithiques, le GH 40 renferme aussi du matériel datable comme des charbons de bois, des os brûlés et des dents de grands herbivores (voir infra).
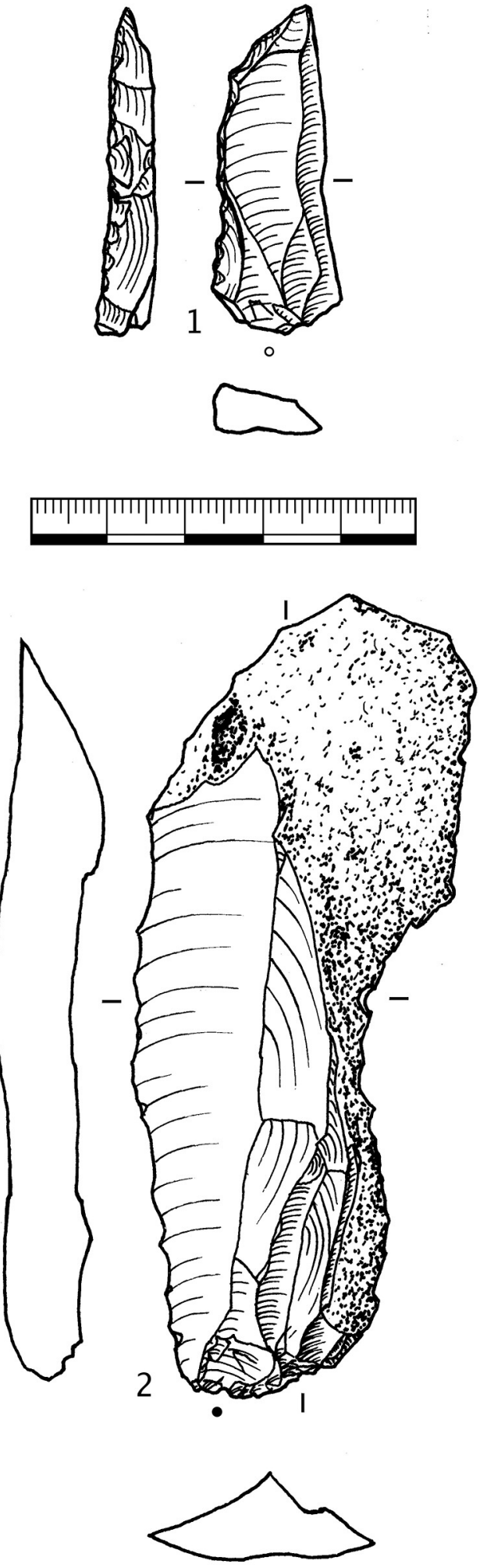

Figure 5 - Grotte de La Verpillière I, fouilles Tübingen, niveau Châtelperronien (GH 40, intact): 1 : pointe de Châtelperron, 2 : lame sous-crête (réalisation : H. Würschem, Ch. Hoyer).

Figure 5 - Grotte de la Verpillière I, Tübingen excavations, Châtelperronian layer (GH 40, intact): 1: Châtelperronian point, 2: secondary crested blade (realization: $H$. Würschem, Ch. Hoyer) 
Stratigraphiquement, le GH 40 est encadré par le niveau inférieur GH 41, subdivisé en plusieurs sous-niveaux. Le GH 41 est à distinguer surtout par l'absence de la coloration rougeâtre, mais apparemment caractérisé par la même matrice. Archéologiquement, le GH 41 a fourni un total de 434 artefacts lithiques. Au niveau techno-typologique, la série est attribuable au Paléolithique moyen avec présence du concept Levallois. On note la présence d'éclats Levallois, d'encoches ainsi qu'un racloir. Le sous-niveau GH 41d contient un élément bifacial. Au-delà, nous signalons une dent, des charbons de bois ainsi que des os brûlés, fragmentés et digérés par les carnivores. En contrebas, se situe un niveau peu épais (GH 15b), se caractérisant par une industrie Paléolithique moyen et s'étendant latéralement en direction de l'ouest, vers les niveaux GH 16/15. À l'origine, en position supérieure du $\mathrm{GH} 40$, se situait un Aurignacien, coincé entre deux tranchées de Charles Méray (fig. 2 et fig. 3). Déstabilisé, ce niveau (GH 15c) a glissé vers la base de l'ancienne tranchée (fig. 3), (Bachellerie et al. 2011).

En 2015, nous avons réalisé, à l'aide d'une pelle mécanique, une grande tranchée (sondage 2) d'une longueur de $25 \mathrm{~m}$, subdivisée en 10 zones. Ce sondage s'étend de l'entrée de La Verpillière I jusqu'à la route en bas du talus. D'un point de vue stratigraphique, la tranchée a été poursuivie jusqu'à la roche-mère. Cette base de la stratigraphie représente plus ou moins la paléo-surface pendant l'occupation paléolithique. Dans sa partie supérieure, la tranchée montre une terrasse d'une largeur de sept mètres. D'une orientation presque horizontale, elle fournit des conditions idéales pour une occupation par l'homme. En contrebas de cette terrasse, la roche apparaît systématiquement délitée en marches, formant comme un escalier qui descend vers le fond de la vallée.

Dans la partie centrale de la terrasse, à environ cinq mètres de l'entrée de la grotte I, se sont rencontrés, au-dessus du calcaire en place, des dépôts non perturbés caractérisés par une accumulation très dense d'esquilles d'os, d'os calcinés et de silex, intégrés dans une situation de piège sédimentaire, dans un milieu d'argile sableuse de couleur jaune. Le corpus de ce niveau d'une épaisseur de $10 \mathrm{~cm}$ a été, vu sa richesse archéologique, fouillé manuellement. En dessous du niveau humique de surface $(\mathrm{GH} 1)$, la fouille a permis d'identifier trois niveaux géologiques, les GH's 44 , 44b et 45 , dans lesquels on distingue quelques accumulations d'artefacts. L'industrie lithique est particulièrement abondante et elle s'élève approximativement à plusieurs milliers de pièces. On y trouve des pièces de la fin du Paléolithique moyen jusqu'à l'Aurignacien. Notons dans ce contexte quelques éléments du Châtelperronien. II s'agit surtout de plusieurs pointes de Châtelperron (fig. 6.7 ; fig. 8.11, fig. 9.3).

\section{Le mobilier châtelperronien}

Dans le cadre de cette publication, nous tenons à présenter la totalité des vestiges attribuables, avec une certaine probabilité, au Châtelperronien. Ce travail comprend des pièces pour la plupart issues des fouilles anciennes et de sédiments fouillés récemment, mais aussi provenant des remblais des fouilles anciennes. II y a aussi quelques éléments stratifiés. Étant donné qu'il n'y a aucun argument pour présenter ces vestiges subdivisés en séries, lieux ou niveaux, nous les présentons ensemble. Une bonne partie de ces objets a été sélectionnée suivant des critères typologiques. Notre objectif, au-delà de la présentation des fossiles-directeurs, est de présenter également des éléments technologiques (nucléus, débitage), appartenant probablement au Châtelperronien (voir Würschem 2015).

\section{Les pointes de Châtelperron}

La grotte de La Verpillière I a donné à ce jour 47 pointes de Châtelperron (fig. 6-9). Deux exemplaires supplémentaires proviennent de la grotte II, ce qui fait un total de 49 pointes. Seules les pièces de La Verpillière I ont été intégrées dans les analyses. Vingt-sept pointes proviennent des fouilles anciennes dont 17 de la toute première de Charles Méray (1868), sept de la fouille et des ramassages Gros (1951-1952), une seule pièce de la fouille Delporte (1953-1955), une pointe est issue des ramassages Thevenot (1956-1957) et une autre d'une fouille non-définie (Dutkiewicz 2011, Dutkiewicz \& Floss 2015). Vingt pointes proviennent des fouilles récentes $(\mathrm{GH} 1,19,40,44 \mathrm{~B}$ et $44 \mathrm{X})$. L'analyse lithique emprunte le système de Tübingen (Hahn 1993 ; Floss 2012) tout en adoptant quelques éléments d'autres études, nous pensons notamment à celle de La Grande Roche de la Plématrie à Quinçay (Vienne) (Roussel 2011, 2013, 2014 ; Roussel \& Soressi 2010 ; Roussel, Soressi et Hublin 2016), à l'étude du matériel de la grotte du Renne à Arcy-sur-Cure (Connet 2002) ainsi qu'à celle de Roc-de-Combe et de La Côte (Pelegrin 1995).

\section{Matières premières}

En ce qui concerne les matières premières siliceuses des pointes de Châtelperron de Germolles, la plupart des pièces ( $n=38,80,85 \%$ ) sont réalisées en silex issu des argiles à silex locaux de la Côte Chalonnaise. Tandis que pour le débitage de ce site, tout au long de la séquence du Paléolithique supérieur, le taux de ce type de silex local s'élève approximativement à $95 \%$. Pour les pointes de Châtelperron le taux des matières non-locales est donc plus élevé $(19,15 \%)$. Deux pointes sont en chaille rose, quatre en silex indéterminé et trois en silex tertiaire (fig. 8.5, fig. $9.1,12)$ zoné très probablement provenant des affleurements du Mont-lès-Étrelles en Haute-Saône, à 120 km nord-est de Germolles (Floss 2003) et apportées sur place sous forme de pointes finies. Cette hypothèse est soutenue par l'absence de nucléus et de débitage châtelperronien pour cette matière première. Cette provenance renvoie à une extension géographique orientale peu connue du Châtelperronien et ravive également la question du Châtelperronien du Trou de la Mère Clochette (voir infra).

\section{Dimensions}

La largeur moyenne des pointes de Châtelperron (fig. $10 \mathrm{a}$ ) est de $1,76 \mathrm{~cm} \pm 0,34$, l'épaisseur moyenne (fig. $10 \mathrm{~b}$ ) de $0,67 \mathrm{~cm} \pm 0,19$ et la longueur moyenne (fig. $10 \mathrm{c}$ ) des pièces complètes $(n=24)$ est de $5,55 \mathrm{~cm} \pm 1,36$. La pointe 

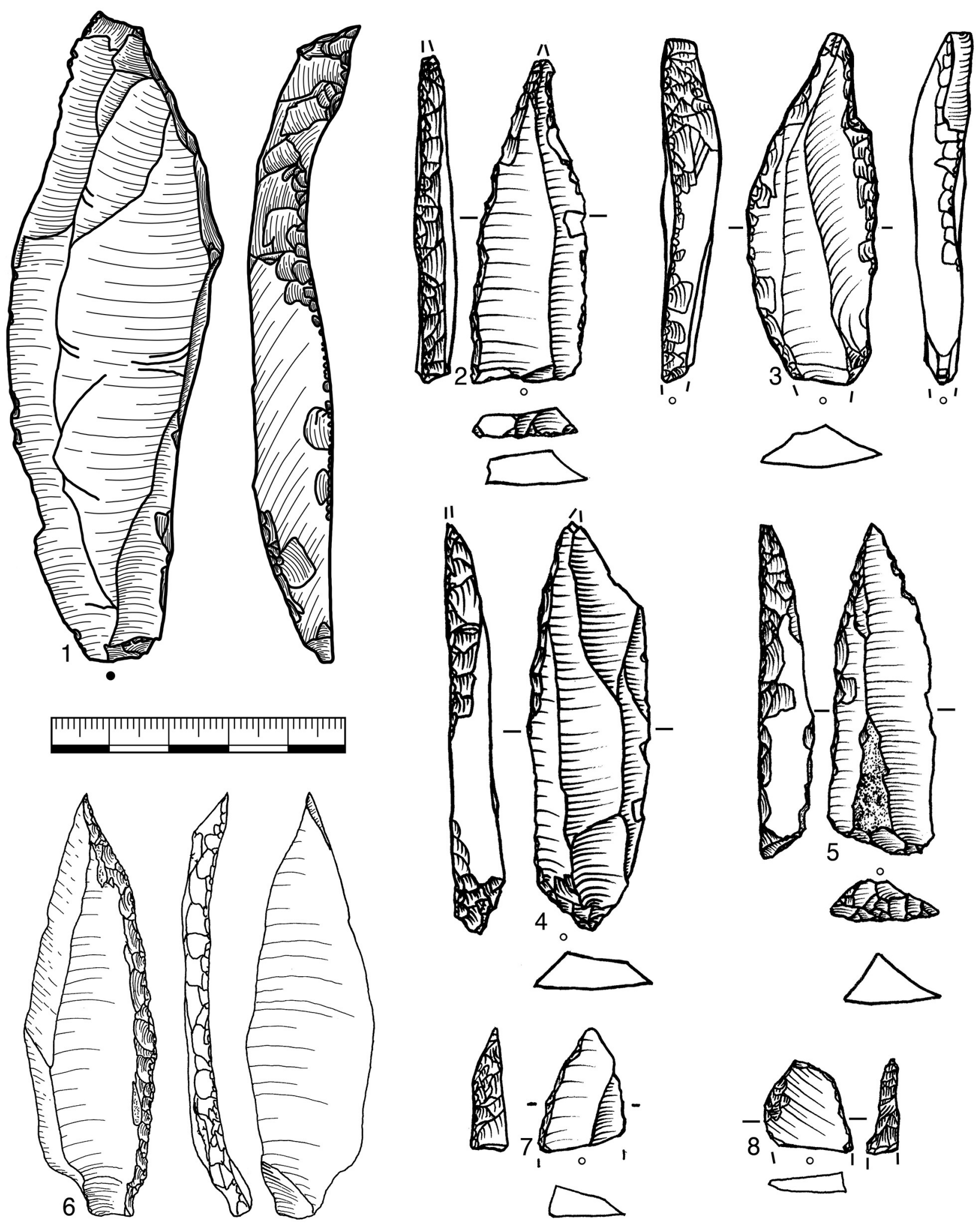

Figure 6 - Grotte de La Verpillière I, pointes de Châtelperron. 1, 3-5 : fouilles Méray, 2 : sondages Gros, 6-8 : fouilles Tübingen (réalisation : H. Würschem, Ch. Hoyer).

Figure 6 - Grotte de la Verpillière I, Châtelperronian points. 1, 3-5: Méray excavations, 2: sondages by Gros, 6-8: Tübingen excavations (realization: H. Würschem, Ch. Hoyer). 

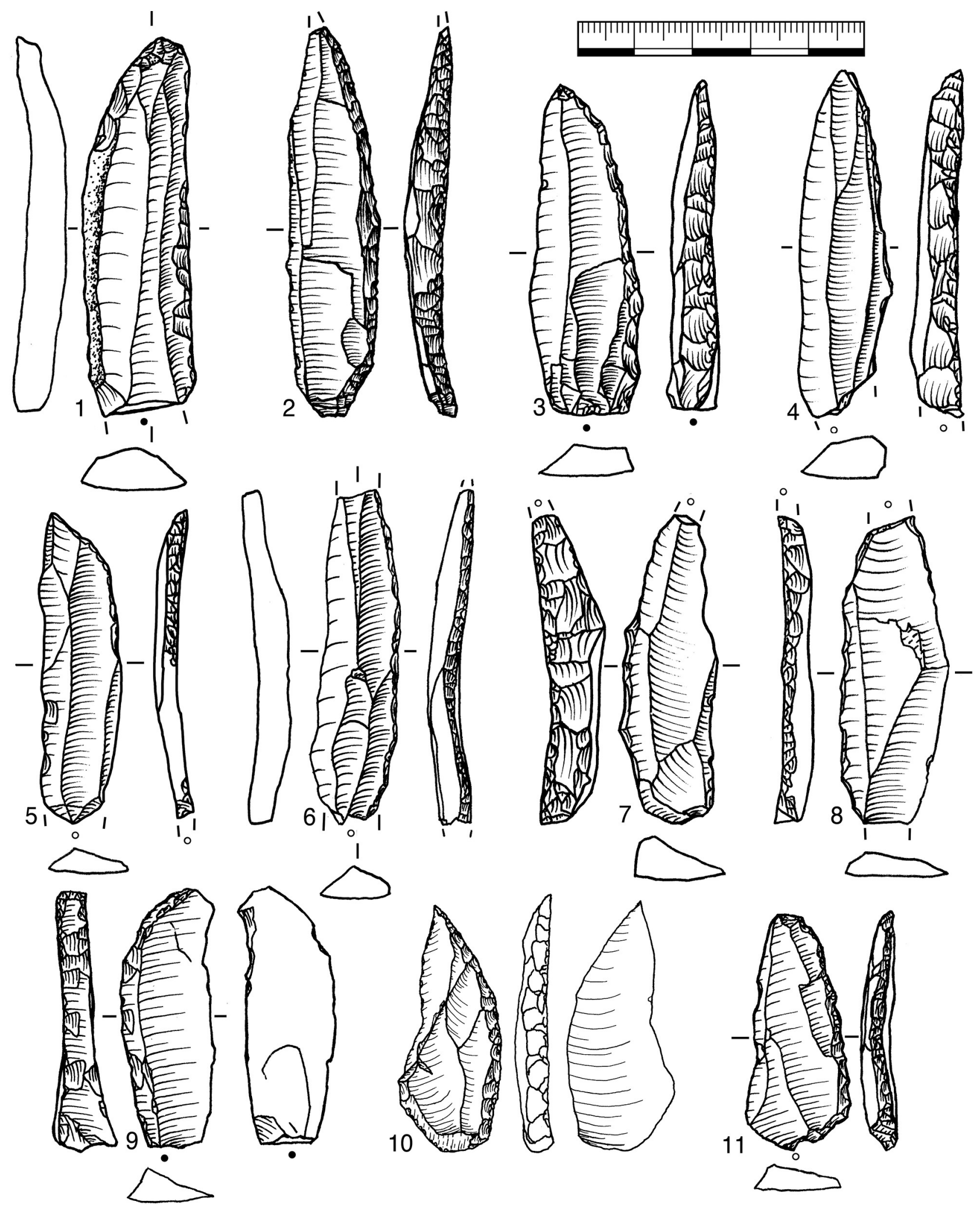

Figure 7 - Grotte de La Verpillière I, pointes de Châtelperron. 1, 2, 4-6, 8 : fouilles Méray, 3, 7 : sondages Gros, 10 - 12 : fouilles Tübingen (réalisation : H. Würschem, Ch. Hoyer).

Figure 7 - Grotte de la Verpillière I, Châtelperronian points. 1, 2, 4-6, 8: Méray excavations, 3, 7: sondages by Gros, 9-11 Tübingen excavations (realization: $\mathrm{H}$. Würschem, Ch. Hoyer). 

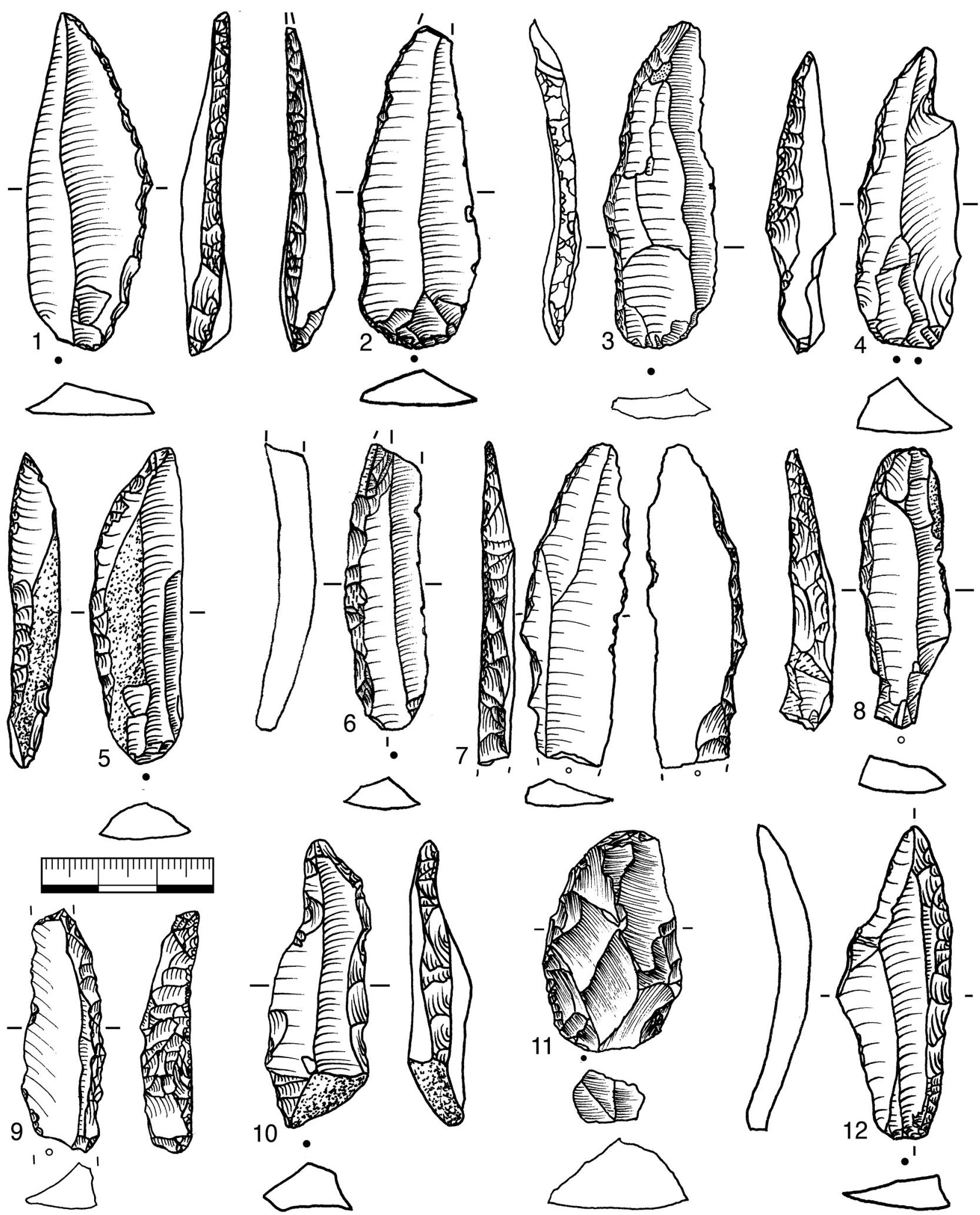

Figure 8 - Grotte de La Verpillière I, pointes de Châtelperron. 1, 2, 4-6, 11 : fouilles Méray, 9 : sondages Gros, 8 : fouille indéterminée, 3 , 7, 10 : fouilles Tübingen (réalisation : H. Würschem, Ch. Hoyer).

Figure 8 - Grotte de la Verpillière I, Châtelperronian points. 1, 2, 4-6, 11: Méray excavations, 9: sondages by Gros, 8: unknown excavator, 3, 7, 10: Tübingen excavations (realization: H. Würschem, Ch. Hoyer). 

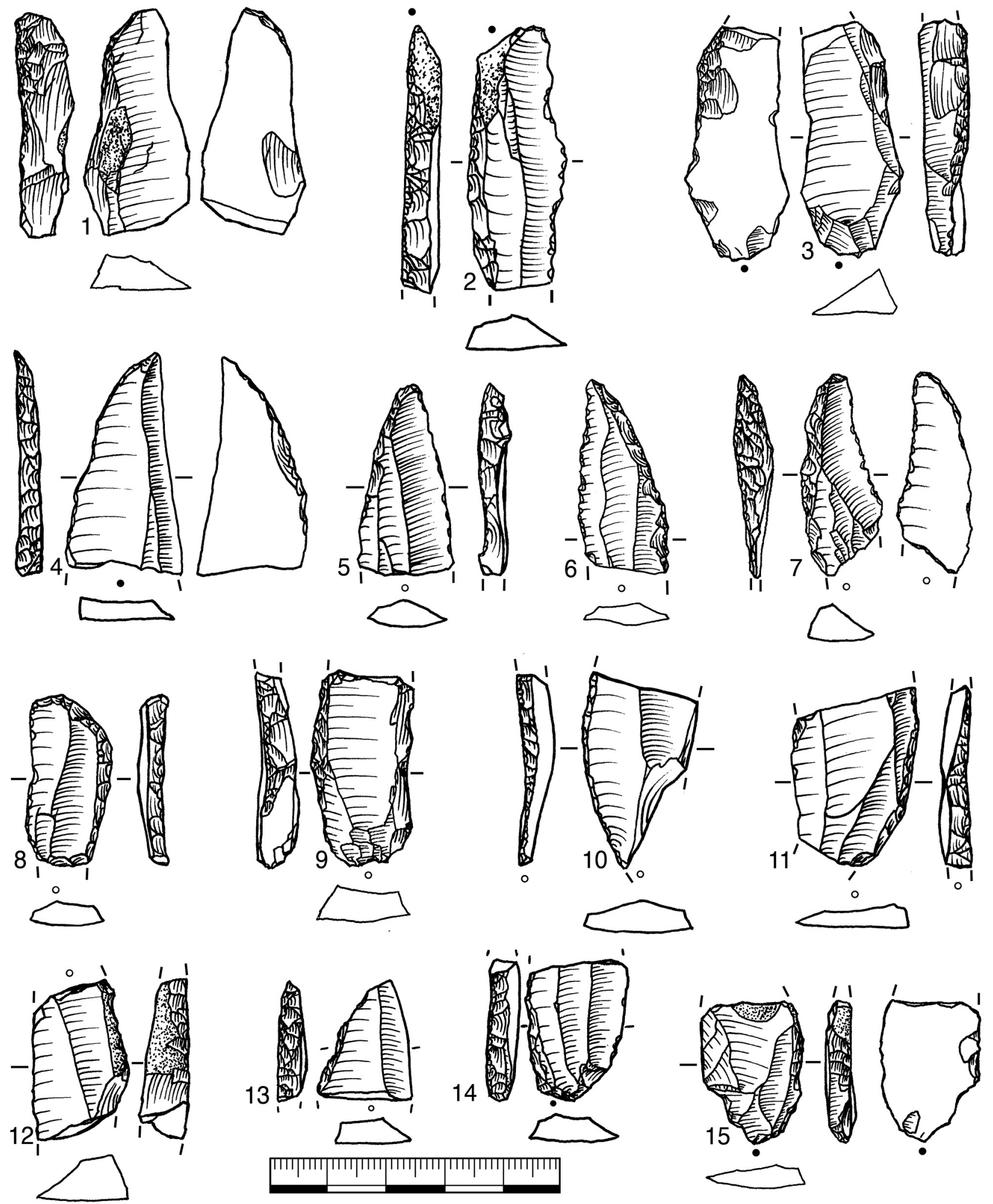

Figure 9 - Grottes de La Verpillière I, pointes de Châtelperron. 1, 3, 6, 7, 9, 12, 13 : fouilles Tübingen, 2 : fouilles Méray, 5, 8, 11 : sondages Gros, 4 : fouilles Delporte, 10 : sondage Thevenot (réalisation : H. Würschem, Ch. Hoyer).

Figure 9 - Grotte de la Verpillière I, Châtelperronian points. 1, 3, 6, 7, 9, 12, 13: Tübingen excavations, 2: Méray excavations, 5, 8, 11: sondages by Gros, 4: Delporte excavations, 10: Thevenot sondage (realization: H. Würschem, Ch. Hoyer). 
la plus longue (fig. 6.1) mesure $10,73 \mathrm{~cm}$, mais elle représente un cas exceptionnel. Les dimensions trouvent un bon équivalent à Quinçay où les longueurs des pointes mesurent $5,18 \mathrm{~cm} \pm 1,39$ pour le niveau châtelperronien inférieur En, $5,42 \mathrm{~cm} \pm 1,31$ pour le niveau moyen Em et de $4,38 \mathrm{~cm} \pm, 081$ pour le niveau supérieur $\mathrm{Ej}$. La largeur pour ces niveaux est à $1,56 \mathrm{~mm} \pm 0,38 ; 1,69 \mathrm{~cm} \pm 0,39$; et $1,39 \mathrm{~cm} \pm 0,26$ (Roussel, Soressi et Hublin 2016). D. Baffier (1999) définit comme longueur typique 4 à $6,5 \mathrm{~cm}$, en indiquant une valeur extrême de $10 \mathrm{~cm}$. À Roc-de-Combe, 52 des 84 pointes de Châtelperron complètes mesurent en longueur 4,0 à 6,0 cm (Pelegrin 1995).

L'allongement (relation longueur-largeur) des pointes de Châtelperron complètes de Germolles est de 2,99 $\pm 0,50$ et la robustesse (relation largeur-épaisseur) de $2,82 \pm 0,86$. À Quinçay, ces indices sont à $3,4 \pm 0,8$ et $3,1 \pm 0,9$ (niveau En), à 3,3 $\pm 0,6$ et 3,4 $\pm 0,9$ (niveau Em) et à 3,2 \pm 0,5 et 3,2 $\pm 0,7$ (niveau Ej) (Roussel, Soressi et Hublin 2016). À Quinçay a été également prise la rectitude du dos, c'est-à-dire "sur les pièces entières la portion rectiligne du dos strictement perpendiculaire à la base de l'objet " (Roussel 2011 - p. 145). Ce procédé a été appliqué pour tester l'hypothèse de $F$. Lévêque d'après laquelle les pointes du Châtelperronien " évolué " (niveau Em) seraient plus redressées que celles du Châtelperronien " ancien ou typique » (niveau En) (Roussel \& Soressi 2010 ; Roussel 2011 ; Roussel, Soressi et Hublin 2016). À la grotte de La Verpillière I, sans subdivision en plusieurs niveaux, nous n'avons pas effectué ce type de mesure. Le poids moyen des pointes de Germolles, mesuré sur 10 pièces, s'élève à $6,6 \mathrm{~g}$.

\section{Débitage et orientation}

Les supports des pointes de Châtelperron sont tous des lames. Elles proviennent le plus souvent d'un débitage unidirectionnel $(78,72 \%)$, comme cela a été observé à Arcy-sur-Cure (Connet 2002), mais dix lames portent les stigmates d'un débitage bidirectionnel $(21,28 \%)$. En ce qui concerne la technique de taille, on observe une grande variabilité. Tandis que quatre supports des pointes ont été visiblement débités par percuteur dur, huit montrent des stigmates de la percussion tendre directe et même sept de la pierre tendre. Ces observations se différencient de celles faites à Roc-de-Combe et à La Côte mettant en évidence prioritairement la percussion tendre directe (Pelegrin 1995, mais corrigé en pierre tendre dans Pelegrin 2012).

Vingt-deux des 47 pointes ont été réalisées sur des lames de section asymétrique (Roussel 2011) qui ont facilité leur aménagement. On distingue des lames à dos s'appuyant sur un pan cortical abrupt $(n=4)$ ou sur une crête ou néocrête $(n=6)$ ou encore sur un pan abrupt formé d'un négatif en position orthogonale vis-à-vis des autres enlèvements dorsaux $(n=12)$. À Quinçay, un cinquième des pointes représente cette dernière configuration (Roussel, Soressi et Hublin 2016 - p. 17).

Quant à l'orientation de la pointe active en relation avec le support, sur cinq pièces $(10,6 \%$ ) la pointe se situe sur l'extrémité proximale et non distale de la lame. À Arcy-sur-
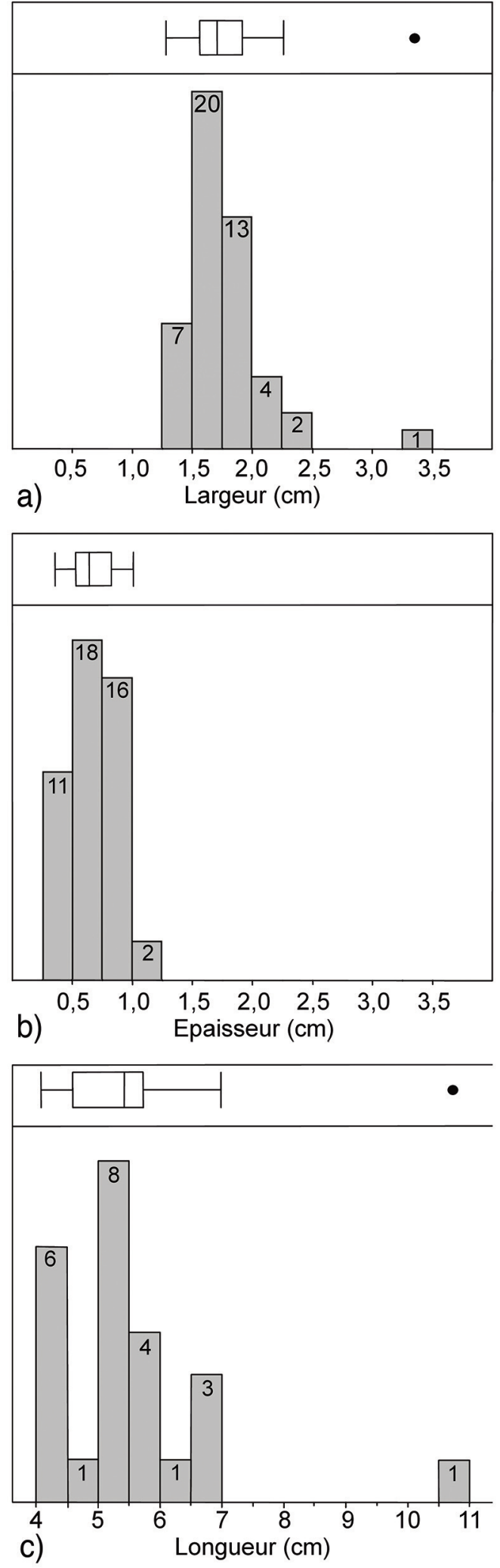

Figure 10 - Dimensions des pointes de Châtelperron. a) largeur, b) épaisseur, c) longueur des pièces complètes.

Figure 10 - dimensions of the Châtelperronian points. a) width, b) thickness, c) length of the complete artefacts. 
Cure, N. Connet observe, suivant les niveaux, 5 à $15 \%$ de ce genre de pièces : "Cette inversion est observée lorsque la morphologie de la partie distale du support ne permet pas l'aménagement de la pointe, lorsqu'elle est trop épaisse, outrepassée, ou présente un profil trop courbe ou encore lorsqu'elle est trop fine, ne permettant pas la mise en place de la courbure de la pointe recherchée. " (Connet 2002 - p. 370). À Quinçay, le taux de cette inversion s'élève à 10-15\% (Roussel 2011).

\section{Latéralisation}

Dans quelques séries châtelperroniennes, comme à Pairnon-Pair ou à Brassempouy, on observe l'aménagement du dos abattu des pointes de préférence sur le côté droit de la pièce (Baffier 1999 - p. 60-62). À La Verpillière I, c'est différent. Le dos se trouve pour 27 pièces $(57,4 \%)$ à gauche et pour $20(42,65 \%)$ à droite. À Quinçay, cette relation est pour le niveau En à $52 \%$ droite/ $48 \%$ gauche, au niveau Em 45\% droite/ 55\% gauche et dans le niveau supérieur Ej à $75 \%$ droite/ $25 \%$ gauche, en sachant que ce dernier niveau ne compte que huit pièces (Roussel 2011). À Arcy-sur-Cure, le résultat est similaire : "Le dos se situe indifféremment à gauche ou à droite des supports " (Connet 2002 - p. 370). Toujours à Arcy, le dos est formé par des retouches directes, larges et régulières. "Dans de rares cas, la retouche est croisée, notamment lorsque le dos est trop abrupt par rapport à la face d'éclatement du support, ce qui intervient surtout dans le dernier tiers de l'outil, vers la pointe » (Connet 2002 - p. 369). Cette même observation vaut pour plusieurs pièces de Germolles (par exemple fig. 6.2, 7.7 ou 9.7). À Quinçay, on connaît ce même phénomène où ce type de retouche accentue le caractère déjeté de la pointe et permet la réalisation plus aisée de l'épaisseur voulue du dos (Roussel 2011 - p. 156).

\section{Traces d'impact}

La littérature d'archéologie expérimentale décrit des stigmates localisés sur la pointe de projectiles interprétés comme traces d'impact, ils se retrouvent également sur certaines pointes de Châtelperron (Pelegrin et Soressi 2007, 288). On y distingue de simples fractures, des fractures esquillantes et des fractures en cône qui s'initient d'une fracture en flexion, enlevant un petit éclat ou une esquille (Fischer, Vemming Hansen 1984 ; O'Farrell 2004 ; O'Farrell 2005 - p. 398). Nous pouvons y ajouter des traces d'impact de type pseudo-burin et fractures écrasées (crushing) montrant un „cluster of overlapping step scars parallel to the long axis of the tool" (Villa et al. 2009 - p. 855). Ce sont les pointes de Châtelperron qui sont souvent interprétées comme pointes de projectile dans le contexte du Châtelperronien. L'argumentation pour cette attribution repose sur les «apical shock stigmata». Les quatre pointes de Châtelperron de Brassempouy (Abri Dubalen, Landes) (Bachellerie et al. 2011 - p. 138) fracturées et les deux pointes portant des traces d'impact de Labeko Koba (RiosGaraizar 2008 - p. 28) en sont des exemples.

À Germolles, 11 pointes de Châtelperron portent des stigmates identifiés comme traces d'impacts. On compte sept fractures en cône, un " crushing " et trois pseudoburins (fig. 11).

\section{Autres éléments retouchés}

D'autres types d'outils lithiques comme par exemple les grattoirs châtelperroniens, grattoirs « (...) sur large éclat épais présentant un front de grattoir plutôt étendu » (Demars et Laurent 1989 - p. 154) ou sur " éclats envahissants laminaires 》 (Roussel 2011 - p. 108) (voir aussi (Roussel 2011 ; Rios-Garaizar et al. 2012 ; Baillet, Bachellerie et Bordes 2014 ; Roussel, Soressi et Hublin 2016) et équipés « soit des négatifs laminaires parallèles ou subparallèles (...), soit des négatifs d'éclats envahissants antérieurs (...) 》 (Roussel 2011 - p. 108), n'ont pas été découverts à Germolles, mais nous n'excluons pas leur présence après un tri encore plus minutieux du grand lot d'éclats de ce site.

\section{Couteaux de type Abri Audi}

Les couteaux de type Abri Audi se rencontrent dès le Moustérien de tradition acheuléenne, mais ils apparaissent aussi, en petite quantité, dans le Châtelperronien (Bordes 1953 ; Sonneville-Bordes et Perrot 1956 ; Roussel 2011 ; Bolus 2012). À Germolles, trois pièces satisfont aux conditions de définition de ces couteaux (fig. 12.1,2,5) et nous y ajoutons trois supports non retouchés avec un dos naturel (fig. 12.3, 4, 6).

\section{Nucléus}

Au-delà des fossiles-directeurs et de leurs composantes emblématiques, les pointes de Châtelperron, nous insistons sur la question de la présence d'un débitage sur place. Le nombre important de pointes connues $(n=44)$, qui n'est certainement qu'une petite partie de l'ancien total pour ce site, plaide en faveur d'une occupation châtelperronienne plutôt consistante. Néanmoins, la mise en évidence du débitage fortifie l'aspect de l'implantation humaine sur place. Issu largement d'un contexte remanié, il a fallu entreprendre une étude comparative avec des séries contemporaines, de préférence de Bourgogne (Grotte du Renne, Connet 2002), mais aussi de l'ouest du Massif central (Quinçay, Roussel 2013 ; Roc-de-Combe et La Côte, Pelegrin 1995).

À Quinçay, M. Roussel définit trois groupes de nucléus, les nucléus exploités sur deux faces, sur trois faces et sur une seule face étroite. La particularité repose sur un "rythme de débitage en deux temps (...) sur table laminaire de section anguleuse »(Roussel 2013 - p. 236) débutant depuis une crête et s'étendant d'abord sur une face et après sur l'autre. Les faces sont exploitées en déconnexion les unes des autres, constituant chacune une table de débitage indépendante de la précédente (Roussel, Soressi et Hublin 2016)

Nelly Connet définit cinq groupes de nucléus y compris de nombreux sous-groupes, qui se distinguent par la construction de leurs volumes et la finalité des supports. Les groupes I, II et III sont particulièrement importants pour 


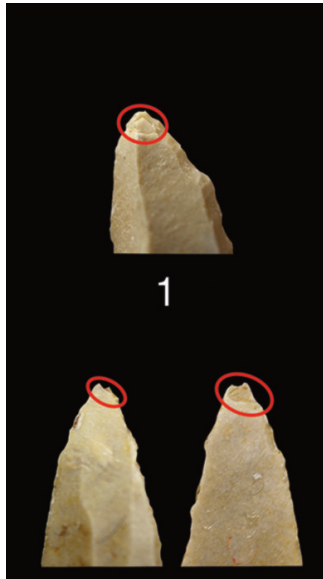

4

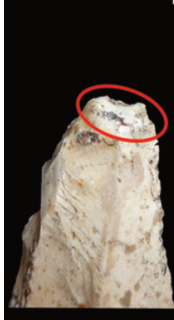

7

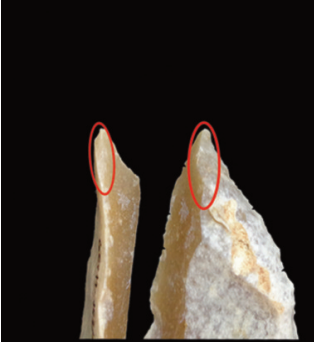

10

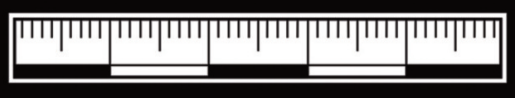

11

2

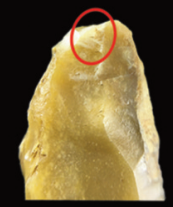

5

8

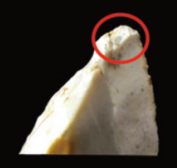

3

6
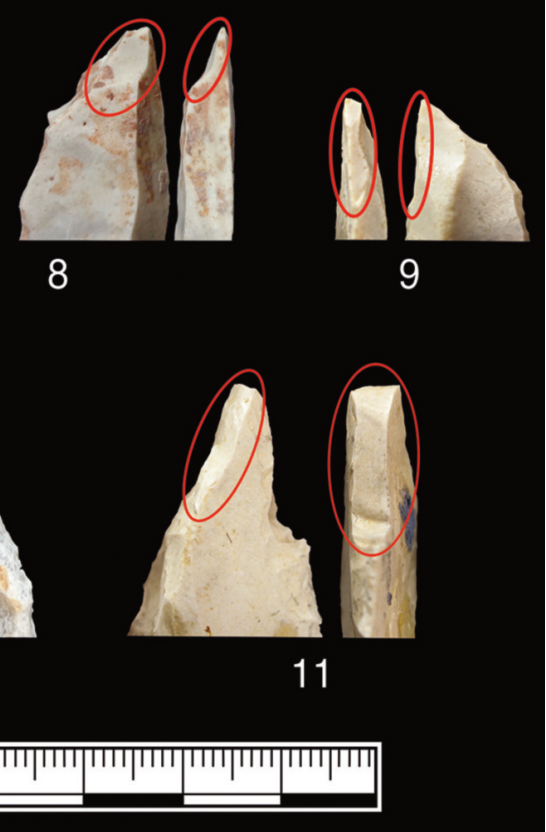

Figure 11 - Grotte de La Verpillière I, traces d'impacts sur les extrémités distales actives des pointes de Châtelperron. 1 : crushing, 2-8: fractures en cône, 9-11: pseudo-burins (réalisation : H. Würschem, Ch. Hoyer).

Figure 11 - Grotte de la Verpillière I, impact scars on the active distal ends of Châtelperronian points. 1: crushing, 2-8: cone fracturing, 9-11: pseudo-burin fracturing (realization: H. Würschem, Ch. Hoyer).

la production de lames châtelperroniennes. Le groupe I comprend des nucléus à lames, uni- ou bidirectionnels, disposant d'une crête longitudinale, aménagée ou naturelle. Les flancs du nucléus sont préparés ou non. L'exploitation est initiée à partir des crêtes et a lieu sur la face principale, mais peut aussi déborder sur les flancs (Connet 2002 - p. 40-42). Le groupe II comprend également des nucléus à lames uni- ou bidirectionnels, mais l'exploitation laminaire dépasse les flancs et envahit les trois quarts de la périphérie du nucléus. Le groupe III comprend également des nucléus à lames uni- ou bidirectionnels mais dispose de deux flancs attenants. Le premier est le dos du nucléus et il est non- ou peu préparé. Le deuxième est la face d'exploitation, mais elle n'est que peu débitée. On ne trouve que rarement le cas où seules les deux faces sont débitées.

Jacques Pelegrin distingue sept types de nucléus (1995 p. 115-116). En premier lieu, il distingue les nucléus qui sont débités sur face étroite, sur face large ou sur faces étroite et large. En second lieu, il prend en considération des plans de frappe. Le groupe 1 contient donc des nucléus avec deux plans de frappe opposés. L'exploitation couvre la partie la plus étroite de la partie transversale du nucléus. Ce nucléus a une allure prismatique. Le groupe 2 réunit des nucléus avec deux plans de frappe opposés. L'exploitation couvre de préférence la partie la plus large de la partie transversale du nucléus. Ce nucléus a une allure plutôt prismatique, quand il est très large, il est plutôt plat. Le groupe 3 représente des nucléus avec deux plans de frappe opposés. L'exploitation a lieu sur une face étroite et, par la suite, sur une face large. Ce nucléus a une allure plutôt prismatique. Les groupes 4,5 et 6 ressemblent aux groupes décrits auparavant, mais ne disposent pas d'un plan de frappe. Le groupe 7 de Roc-de-Combe est rare et comprend des nucléus avec deux plans de frappe opposés et avec deux surfaces de débitage indépendantes.

Contrairement à la production de lames, au sein du Châtelperronien la production d'éclats ne joue qu'un rôle subordonné à la production de lames, ou en tout cas très secondaire. À Quincay, parmi les 452 nucléus étudiés, 363 sont des nucléus à lames, 51 des nucléus à lamelles et seulement neuf sont considérés comme de nucléus à éclats indépendants (Roussel, Soressi et Hublin 2016). À la grotte du Renne à Arcy-sur-Cure, le taux de nucléus à éclats s'élève entre 16 et $23 \%$, décroissant en direction des niveaux supérieurs. II y a des nucléus centripètes plats unifaciaux et des nucléus discoïdes (Connet 2002 - p. 387). À Germolles, aucun nucléus châtelperronien à éclats n'a été trouvé en place.

$\mathrm{Au}$ total, nous avons identifié 11 nucléus répondant aux critères décrits ci-dessus pour les nucléus châtelperroniens. D'abord, nous distinguons deux nucléus (fig. 13.1,11) avec seulement un plan de frappe dont l'exploitation principale a eu lieu sur la face large de la pièce (Pelegrin groupe 5 , nucléus sur une face d'après Roussel). D'autres nucléus suivent ce même principe (fig. 13.3, 5, 7) mais disposent de deux plans de frappe (Pelegrin groupe 2 ).

On trouve également des nucléus exploités sur trois faces dont deux larges et une étroite (fig. 13. 8), portant le négatif d'un éclat envahissant laminaire. Suivant Roussel (2011 p. 271, fig. 5.31) ils sont dû à une « (...) extraction d'un éclat à peu près aussi large que long sur la face la plus large du volume (...). Dans la plupart de cas, il envahit toute la longueur et la largeur de cette face et il peut même parfois outrepasser ». 

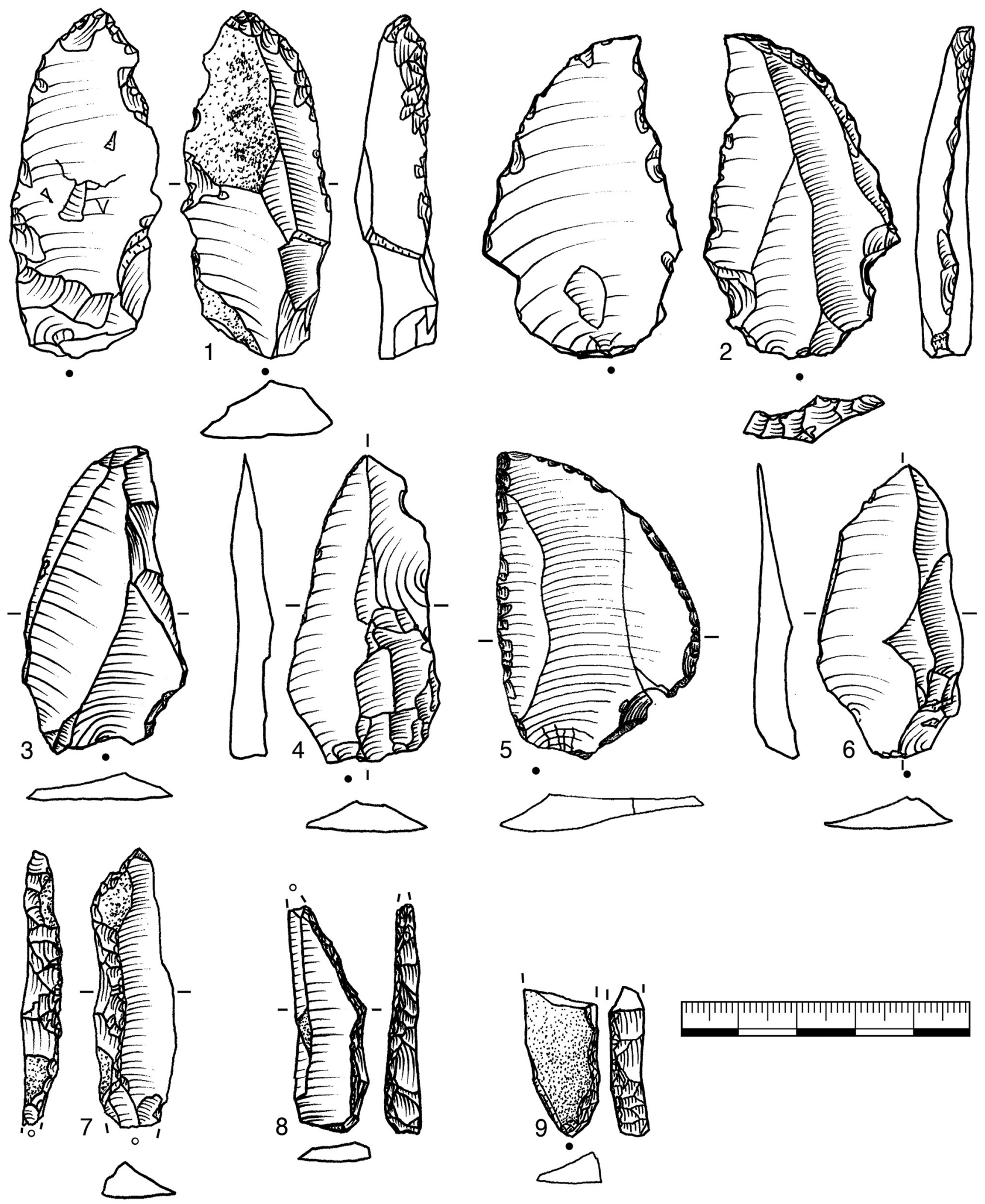

Figure 12 - Grotte de La Verpillière I, couteaux à dos, type Abri Audi: 1 : sondages Gros, 2 : fouilles Méray. 5 : fouilles Tübingen. Éclats oblongs à dos naturel de forme d'un couteau : $3,4,6$ : fouilles Méray, lames à crête ressemblant au pointes de Châtelperron : 7-9: fouilles Tübingen (réalisation : H. Würschem, Ch. Hoyer).

Figure 12 - Grotte de la Verpillière I, backed pieces, type Abri-Audi knife: 1: Gros sondages, 2: Méray excavations, 5: Tübingen excavations. Elongated, naturally backed flakes with the shape of a knife: 3, 4, 6: Méray excavations. Crested blades resembling Châtelperronian points: 7-9 Tübingen excavations (realization: H. Würschem, Ch. Hoyer). 


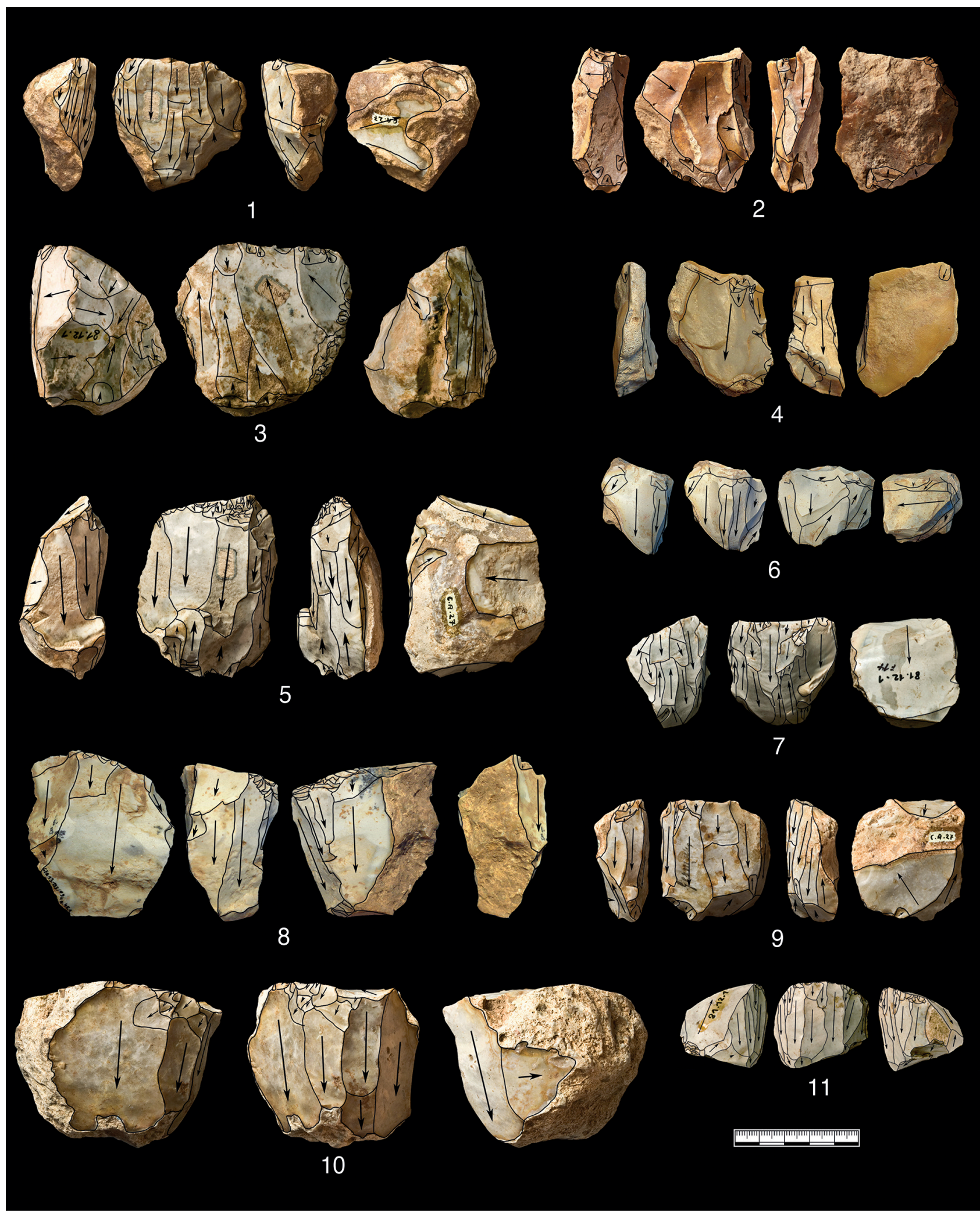

Figure 13 - Grotte de La Verpillière I, Nucléus. 1, 3, 5, 7, 9-11 : fouilles Méray, 2 collection Pelatin, 4, 6, 8 : fouilles Tübingen (réalisation : H. Würschem, Ch. Hoyer).

Figure 13 - Grotte de la Verpillière I, cores. 1, 3, 5, 7, 9-11: Méray excavations, 2: Pelatin collection, 4, 6, 8: Tübingen excavations (realization: H. Würschem, Ch. Hoyer). 
On note également la présence de nucléus plats, disposant d'un plan de frappe, qui ont été exploités sur une face large et puis sur une face étroite (fig. 13.2, 4, 9, 10) (Pelegrin groupe 6 , nucléus à deux faces suivant Roussel). Deux nucléus (fig. 13.2, 4) présentent des restes d'un éclat envahissant laminaire. Ces pièces ressemblent à certaines d'Arcy-sur-Cure (Connet 2002, fig. 30.1), Roc-de-Combe (Pelegrin 1995, fig. 56) et La Grande Roche de la Plématrie (Roussel 2013, fig. 1c).

On y trouve également des nucléus exploités sur une face large et une face étroite, disposant de deux plans de frappe (fig. 13. 6) (Pelegrin groupe 3 , nucléus à deux faces suivant Roussel).

Les nucléus de Germolles sont plus difficiles à comparer à ceux d'Arcy-sur-Cure car ces derniers ont été catégorisés avec de nombreux critères de regroupement. Plusieurs pièces (fig. 13.1, 2, 4) s'apparentent au groupe III, quelques-unes disposant d'une crête latérale au groupe I (fig. 13, 3) et d'autres (fig. 13.6, 7, 10, 11) au groupe II.

Au total, cinq nucléus de Germolles ont été débités sur une face large et cinq autres d'abord sur une face large puis sur une face étroite. Un nucléus a été débité sur trois faces. En ce qui concerne la comparaison avec le regroupement de Pelegrin, trois nucléus de Germolles s'apparentent à son groupe 2 (bidirectionnel, une face), une pièce à son groupe 3 (bidirectionnel, deux faces), deux à son groupe 5 (unidirectionnel, une face) et quatre à son groupe 6 (unidirectionnel, deux faces).

Un nucléus de Germolles en particulier (fig. 13.8) montre des ressemblances avec ce qui a été observé pour les nucléus d'Arcy, de Quincay et les sites de J. Pelegrin notamment à cause de la présence d'un négatif d'éclat envahissant laminaire.

Trois des nucléus figurés (fig. 13.6,7,9,11) sont de petites dimensions et disposent de négatifs laminaires étroits. On ne peut pas identifier une production lamellaire indépendante, proposée par Roussel, Soressi et Hublin pour Quinçay (2016). À Germolles, les nucléus sont fortement exploités et ils sont caractérisés aussi bien par la présence de négatifs lamellaires que laminaires (arguments métriques de la largeur). Aucun nucléus châtelperronien sert uniquement à la production lamellaire.

Parmi les nucléus figurés, quatre pièces disposent de deux plans de frappe (fig. 13.3,5,6 et 7). Pour deux pièces (fig. 13.3 et 7) le deuxième plan de frappe sert de préférence à la correction du volume du nucléus. Un nucléus (fig.13.6) est fortement exploité et il ne permet pas de constater sur une éventuelle production laminaire indépendante depuis le deuxième plan de frappe. Seulement un nucléus (fig. 13.5) semble d'être débité du plan de frappe opposé, avant que la dernière série de lames ne soit détachée depuis le plan de frappe principal. Les autres nucléus ont été débités de manière unidirectionnelle et ne disposent que d'un plan de frappe. À Quinçay, les nucléus à deux plans de frappe sont fréquents. Mais les séries de lames y restent unidirectionnelles, car les deux plans de frappe desservent des surfaces d'exploitation indépendantes (Roussel,
Soressi et Hublin 2016). À Germolles, les nucléus sont trop fortement exploités pour permettre d'identifier un tel type d'exploitation.

\section{Restes de taille bruts}

Nous avons également sélectionné des lames brutes appartenant probablement au corpus du Châtelperronien. Nous nous sommes intéressés de préférence, aux trois groupes de supports définis auparavant comme supports pour les pointes et plus particulièrement aux pièces disposant d'un négatif dorsal d'orientation orthogonal aux autres, type de lame très prisé, car facilitant l'aménagement du dos (Roussel 2013 ; Roussel, Soressi et Hublin 2016). À Quinçay, ce type de lames occupe $20 \%$ des produits laminaires. Il est le produit d'un débitage « à deux temps » ou « à trois temps ", réalisé autour de la transition entre l'exploitation d'une face à l'autre.

Quarante-et-une lames correspondent aux critères définis (fig. 14, 15). Trente-cinq sont en silex local, une pièce est en chaille rosé, quatre sont en silex non déterminés et une pièce est en silex tertiaire. Onze pièces portent un dos en cortex, pour 16 le dos est formé par une crête et 14 lames portent un négatif orthogonal. Les lames retenues disposent d'une largeur moyenne de $1,71 \mathrm{~cm} \pm 0,43$, d'une épaisseur de $0,66 \mathrm{~cm} \pm 0,23$ et d'une longueur (pièces complètes $\mathrm{n}=15$ ) de $4,60 \mathrm{~cm} \pm 1,14$. À Quinçay, les lames mesurent en moyenne $5,1 \mathrm{~cm} \pm 1,5$ en longueur et $1,8 \mathrm{~cm} \pm 0,5$ en largeur (Roussel 2013). L'allongement à Germolles est à $2,68 \pm 0,62$ et la robustesse à $2,79 \pm 1,01$.

À Germolles, il y a trois lames ressemblant (fig. 14,7-9), même sans aménagement du dos par retouche abrupte, par leur forme et par leur asymétrie, parfaitement aux pointes de Châtelperron. Ces pièces sont quasiment des pointes de Châtelperron et elles semblent parfaitement adaptées à l'utilisation, mais nous préférons de ne les pas intégrer dans le corpus des pointes : "De même, la production de lames de section asymétrique permet de nous interroger sur l'utilisation directe de ces produits au Châtelperronien, qui peuvent être considérés fonctionnellement comme des pièces à dos, au même titre que les éclats débordants Levallois qui dans certains cas ont été utilisés bruts " (Roussel 2011 - p. 454, citant Beyries et Boëda 1983).

En ce qui concerne la répartition spatiale des éléments lithiques châtelperroniens (fig. 2), on remarque une étendue assez vaste couvrant aussi bien l'intérieur que la terrasse de la grotte actuelle. Cette observation, elle aussi, plaide en faveur d'une occupation châtelperronienne importante. Les vestiges issus des fouilles récentes ont pu être cartographiés par mètre carré, tandis que les objets issus des fouilles anciennes ne le sont que par couche ou niveau.

\section{Le Châtelperronien en Bourgogne méridionale et en Franche-Comté}

La position géographique de Germolles à la limite orientale de la répartition connue du Châtelperronien (Bosinski 1987 ; Floss 2003 ; Ruebens, Mc Pherron et Hublin 2015) 


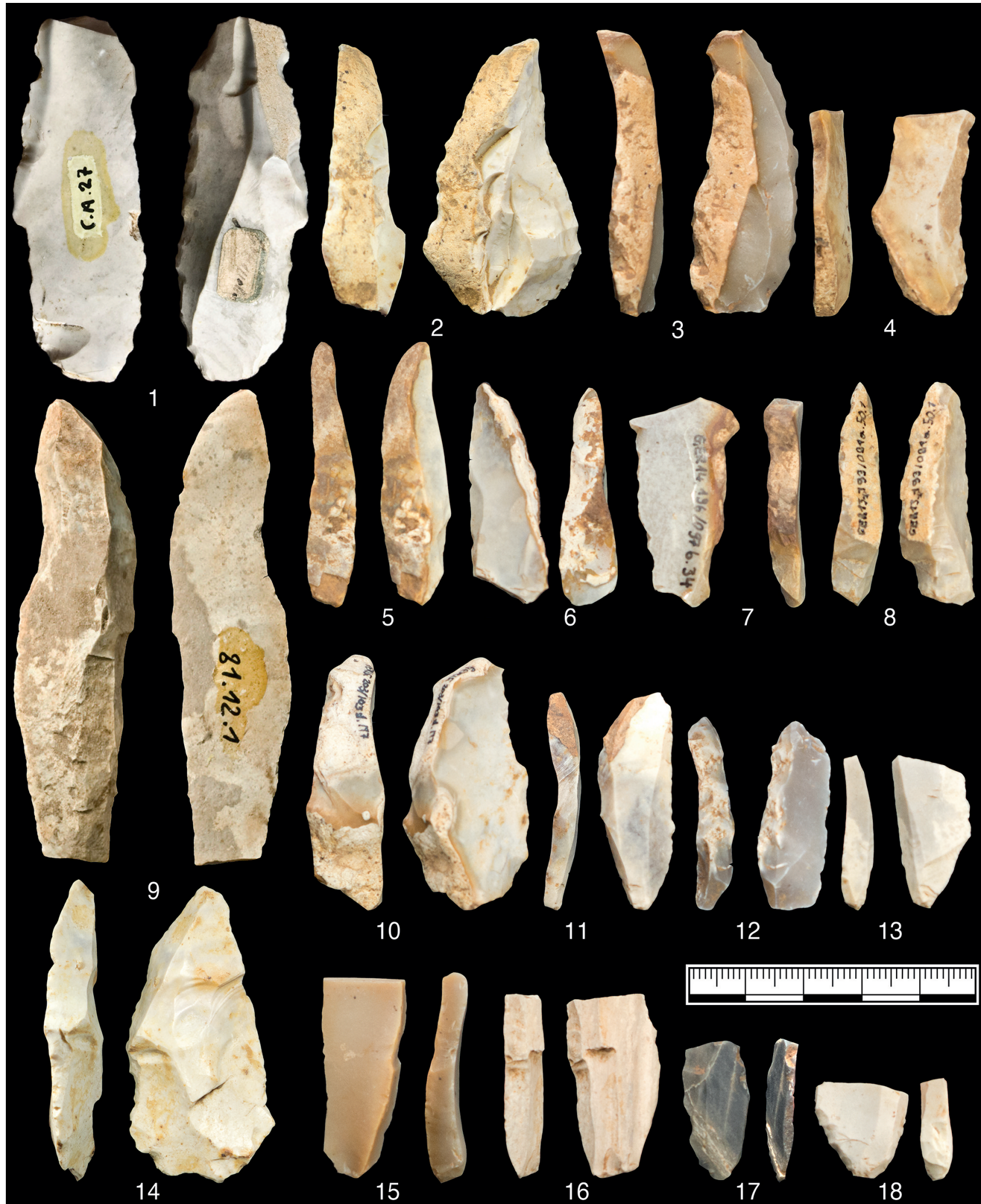

Figure 14 - Grotte de La Verpillière I, lames semi-corticales : 1 : fouilles Méray, 2-12 : fouilles Tübingen. Lames à négatif orthogonal : 9 : fouilles Méray, 13-18 : fouilles Tübingen (réalisation : H. Würschem, Ch. Hoyer).

Figure 14 - Grotte de la Verpillière I, semi-cortical blades: 1: Méray excavations, 2-12: Tübingen excavations. Blades with an orthogonal negative : 9: Méray excavations, 13-18: Tübingen excavations (realization: $\mathrm{H}$. Würschem, Ch. Hoyer). 


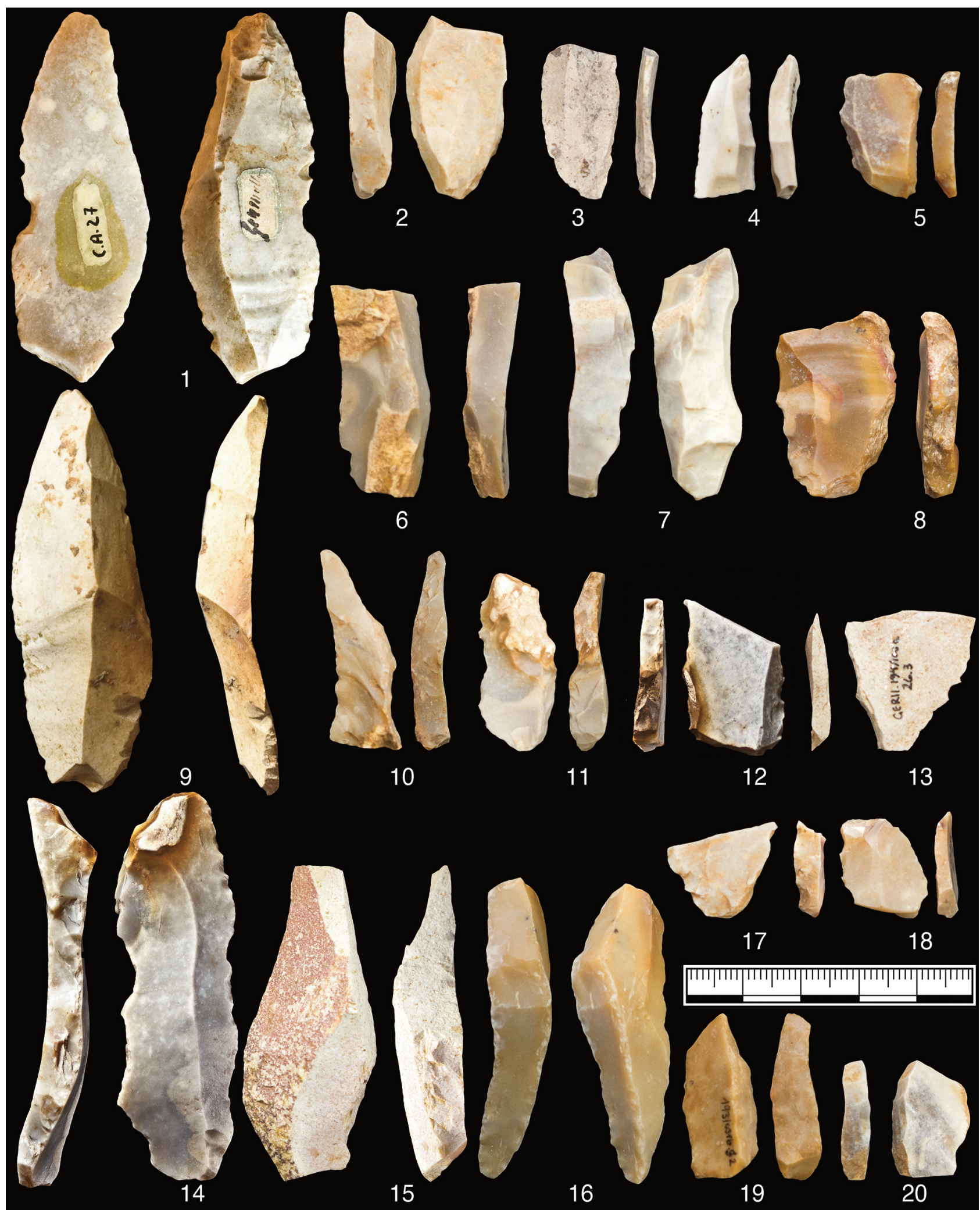

Figure 15 - Grotte de La Verpillière I, Lames à négatif orthogonal : 1, 9 : fouilles Méray, 2-6 : fouilles Tübingen. Lames à crête : 7, 8, 10, 11, 13, 15-20 : fouilles Tübingen, 12, 14 : fouilles Gros (réalisation : H. Würschem, Ch. Hoyer).

Figure 15 - Grotte de la Verpillière I, blades with an orthogonal negative: 1, 9: Méray excavations, 2-6: Tübingen excavations. Crested blades: 7, 8, 10, 11, 13, 15-20: Tübingen excavations, 12, 14: Gros excavations (realization: H. Würschem, Ch. Hoyer). 
crée une position paléogéographique fort intéressante, proche des régions limitrophes, comme par exemple le Jura souabe, où se rencontre un Aurignacien très ancien (Higham et al. 2012). Ceci posé, nous avons réalisé une étude s'intéressant à un possible contexte châtelperronien du site de Germolles dans cette région du bassin de la Saône (voir Floss 2003).

\section{Le Trou de la Mère Clochette}

Le site de loin le plus intéressant à cet égard est le Trou de la Mère Clochette à Rochefort-sur-Nenon (Jura) (R. Desbrosse 1976 - p. 1351), se situant à $85 \mathrm{~km}$ au nord-est de Germolles. Fouillé par J. Feuvrier de 1905 à 1909, et ultérieurement par A. Guichon (Trou aux Renards) et $\mathrm{R}$. Desbrosse, ce site a été, au cours de ces dernières années, surtout investi par L. Brou, s'intéressant à la caractérisation et à la datation de l'Aurignacien (Brou 1997 ; Szmidt, Brou et Jaccottey 2010). Issu des fouilles anciennes, on distingue également une "série jaune ", en bon silex, caractérisée par des dos abattus. Dépourvue d'une stratigraphie convaincante, l'attribution chronologique de cette série repose sur une caractérisation techno- et surtout typologique et hésite entre le Châtelperronien et le Gravettien. Jean Combier (1955 - p. 603) considèra, à l'époque, l'industrie comme typologiquement intermédiaire entre les Périgordiens I et IV de Peyrony, avec des lames à dos « qui mériteraient d'être isolées sous un nom spécial ». R. Desbrosse (1982 - p. 107) se prononce clairement pour l'existence du Périgordien ancien à cause de la présence de nombreux « couteaux à bord abattu » (Desbrosse 1982, fig. 9-11). On n'y compte en fait pas moins de 35 pointes à dos abattu qui ne laissent rien à désirer concernant leur appartenance châtelperronienne (voir aussi Desbrosse 1984 - p. 79). C'est à nouveau J. Combier (1989 - p. 274) qui décrit l'Aurignacien de ce site comme une série mixte avec des éléments à affinités châtelperroniennes. En 1990, ce même auteur (Combier 1990 - p. 270) note la présence " d'une dizaine de couteaux à dos de type Châtelperron incontestable ", mais il maintient l'opinion selon laquelle ces éléments pourraient être incorporés dans l'Aurignacien.

Laurent Brou (Brou 1997 - p. 17) parle d'un « Périgordien au faciès encore mal caractérisé ». La série en question contient 738 éléments lithiques, parmi 361 pièces retouchées. L'auteur est partagé entre une attribution du Châtelperronien évolué et celle d'un Gravettien (Brou 1997 - p. 17). Dans les travaux récents, L. Brou (2009 - p. 70) décrit le site comme une des rares stations de la région témoignant l'existence du Châtelperronien et de l'Aurignacien. Le dilemme repose sur le fait que la série en question, notamment les pointes à dos, sont typologiquement d'allure châtelperronienne et non gravettienne, mais une indication peu claire dans les carnets de fouille de Jules Feuvrier semble indiquer une position stratigraphique de cette série au-dessus de l'Aurignacien (Brou 1997 - p. 19). Nous sommes en mesure d'ajouter un argument : dans la série châtelperronienne de Germolles, y figurent trois pointes réalisées en silex tertiaire du Mont-lèsÉtrelles, affleurement se situant en proximité immédiate du Trou de La Mère Clochette (voir aussi Floss 2003, 280). Cet élément nous fait pencher en faveur d'une série châtelperronienne, ce qui élargirait l'aire de répartition de ce complexe en direction de l'est.

\section{Autres indices châtelperroniens dans la région}

Les autres indices de la présence châtelperronienne de cette région proviennent de la Côte chalonnaise mais sont constitués majoritairement de vestiges isolés.

À Saint Vallerin, à $20 \mathrm{~km}$ au sud de Germolles, juste vers la corniche de la côte, J.-N. Blanchot (inédit, non-daté, p. 20) décrit vaguement une petite série d'artefacts issue d'un canal donnant sur une cavité. Les circonstances de cette découverte restent mystérieuses, mais nous devinons un certain scenario. La série a été soumise à Jean Combier (communication orale 2014) et très brièvement à Harald Floss et nous y soulignons la présence de pointes de Châtelperron.

Chenoves, situé à quelques kilomètres de Saint Vallerin, héberge plusieurs gisements complexes et riches, à proximité des argiles à silex. Les stations sont prospectées depuis l'Abbé Guillard et contiennent quelques éléments châtelperroniens (Guillard 1959 - p.13 ; voir aussi Combier 1959 - p.124) (station Carrouge/ rue Cataux). Au cours de nos études des collections du Musée Denon à Chalon-surSaône, nous avons pu enregistrer une pointe châtelperronienne et un couteau de type Abri Audi (voir aussi Herkert et al. 2015 - p.160).

À Saint Aubin, à quelques kilomètres au nord de Germolles, mais déjà en Côte d'Or, Joly (1959 - p. 98, fig. 7) décrit deux pointes de Châtelperron de l'abri Virely, dont une complète et une fragmentée (voir aussi Floss 2003 - p. 281 ; Floss et al. 2013 b - p-. 336). René Desbrosse (1982 - p. 106, fig. 5) intègre ce site dans le corpus des sites châtelperroniens de l'Est de la France, il reprend ces pièces, les change d'orientation et les dessine différemment. Jean Combier (1990 - p. 270) mentionne, à côté des couteaux à dos courbe, un ensemble correspondant à des lames très étroites et régulières, à dos rectiligne. II rejette cette série comme indice d'un Châtelperronien et l'intègre plutôt dans un Aurignacien (Combier 1990 - p. 277). Récemment, A. Desbrosse et J.-B. Peyrouse sont parvenus à retrouver ces gisements abandonnés.

À Saint-Désert, un peu au sud de Germolles, O. et A.-C. Gros (2005 - p. 169) décrivent, dans la zone entre la Maison-Dieu et le bourg du village, une industrie lithique abondante, parmi laquelle figure, suivant les indications de L. Bonnamour, une pointe de Châtelperron (voir aussi Floss 2003 ; Floss et al. 2013 b - p. 336). Récemment, on a pu retrouver cette pièce dans les collections du Musée Denon à Chalon-sur-Saône.

Un cas particulier est celui du site de Solutré. Jean Combier (1956 ; 1989 - p. 274) mentionne, pour les niveaux profonds, constitués par des cailloutis rouges, par exemple pour la tranchée E de 1922, des couteaux à dos, similaires 
aux pointes de Châtelperron et intègre Solutré, depuis cette publication (1956 - p. 198, 202), dans le corpus du "Périgordien inférieur atypique " (voir aussi Floss et al. 2013 b - p. 335, fig. 2).

Finalement, nous tenons à signaler la grotte de la Folatière à Culles-les-Roches et le site de plein air de Saint-Martinsous-Montaigu, où quelques pièces pourraient relever du Châtelperronien.

Nous en sommes conscients, la plupart des indications sont isolées et sans contexte. Elles ne se rattachent au Châtelperronien que par l'aspect typologique. Néanmoins, Rochefort-sur-Nenon et aussi Saint-Vallerin pourraient représenter des occupations plus importantes. Se précise l'idée que l'occupation châtelperronienne de Germolles ne constitue pas un cas isolé mais s'intègre dans une véritable aire d'occupation châtelperronienne en Bourgogne méridionale.

\section{Remerciements}

Nous tenons à remercier la direction régionale des Affaires culturelles, Service régional d'Archéologie de Bourgogne, I'UMR 6298 ARTeHIS de l'Université de Bourgogne, le Deutsche Forschungsgemeinschaft, le département de la Saône-et-Loire, l'Université de Tübingen, la mairie de Mellecey, les équipes de fouille des grottes de La Verpillière, les membres du PCR «Paléolithique en Bourgogne Méridional " et notamment Pierre Andriot, Laetitia Bassereau-Vauthier, Sabine Boos, Didier Cailhol, Michel Cessot, Jean Combier, René Desbrosse, Victor Donguy, Denis Dubois, Annie Dumont, Ewa Dutkiewicz, Jean-Claude Gress, André-Charles Gros, Nadine Huber, Olivier Lemercier, Stephanie Lismann, Bernard Macioszczyk, Gwenaëlle Marchet-Legendre, Catherine Michel, René Parise, Yves Pautrat, Matthieu Pinette, Michel Prestreau, Benjamin Schürch, François Sikner, Pierre Voarick et Isabelle Vernus.

Et nous tenons à remercier très chaleureusement les deux rapporteurs pour leurs critiques constructives, remarques et corrections.

\section{Références bibliographiques}

ARMAND-CALLIAT L. 1950 - Catalogue des collections archéologiques : menus objets divers des collections orientales et de la salle Niepce. Chalon-sur-Saône : R. Simonin, 100p.

BACHELLERIE F. 2011 - Quelle unité pour le Châtelperronien ? Apport de l'analyse taphonomique et techno-économique des industries lithiques de trois gisements aquitains de plein air: le Basté, Bidart (PyrénéesAtlantiques) et Canaule II (Dordogne). Bordeaux : Université de Bordeaux I, 2011. Thèse de doctorat, 441p.

BACHELLERIE F., BON F., DESCHAMPS M., EIZENBERG L., HENRY-GAMBIER D., MOURRE V., NORMAND CH., PELEGRIN J., PRIMAULT J., SCANDIUZZI R., THIÉBAUT C. 2011 - Archaeological signatures of hunting activities applied to comparisons of Mousterian, Chatelperronian and Aurignacian industries in the Pyrenees: the nature of hunting tools and site functions. In : F. Bon, S. Costamagno, N. Valdeyron (Eds.), Hunting Camps in Prehistory. Current Archaeological Approaches, Proceedings of the International Symposium (may 13-15 2009). Université Toulouse II, Le Mirail, p. 131-167 (Pal@ethnology, №3).

BADER G. 2011 - Stratigraphische Befunde aus dem Paläolithikum der Höhle Grotte de La Verpillière I in Germolles (Saône-et-Loire, Frankreich). Tübingen : Eberhard Karls Universität Tübingen, 2011. Thèse de bachelor, 50p.

BAFFIER D. 1999 - Les derniers Néandertaliens. Le Châtelperronien. Paris : La maison des roches (Histoire de la France préhistorique), 113p.

BAILLET M., BACHELLERIE F., BORDES J.-G. 2014 Enquête autour d'un outil : approche techno-économique, fonctionnelle et expérimentale des grattoirs châtelperroniens de Canaule II (Creysse, Dordogne, France). Paleo, 25, p. 7-36.

BEYRIES S., BOËDA E. 1983 - Étude technologique et traces d'utilisation des 'éclats débordants' de Corbehem (Pas-de-Calais). Bulletin de la Société Préhistorique française, 80, 9, p. 275-279.

BLANCHOT J.-N. non daté - Chenoves, St. Vallerin, le goût Aurignacien. 22p.

BOLUS M. 2012 - Messer mit Rücken. In: FLOSS H. (Ed.), Steinartefakte vom Altpaläolithikum bis in die Neuzeit. Tübingen : Kerns Verlag, p. 287-292.

BORDES F. 1953 - Essai de classification des industries "moustériennes". Bulletin de la Société préhistorique française, 50, 7-8, p. 457-466.

BOSINSKI G. 1987 - Die große Zeit der Eiszeitjäger. Europa zwischen 40.000 und 10.000 v. Chr. 5. Theodor MommsenVorlesung 1986. Mainz, Römisch-Germanisches Zentralmuseum, p. 3-139 (Jahrbuch des RömischGermanischen Zentralmuseums, $\left.n^{\circ} 34\right)$.

BREUIL H. 1909 - L’Aurignacien présolutréen. Épilogue d'une controverse. Revue préhistorique, 4, 8-9, p. 5-46.

BREUIL H. 1911 - Etudes de morphologie paléolithique : I. L'industrie de la grotte de Châtelperron (Allier) et d'autres gisements similaires. Revue de l'Ecole d'Anthropologie, 21, p. $29-40$ et p. 66-76.

BROU L. 1997 - L'industrie aurignacienne du « Trou de la Mère Clochette " à Rochefort-sur-Nenon, Jura. Présentation des données. In : A. Thévenin, A. Villes (Eds.), Le paléolithique supérieur de l'Est de la France : de l'Aurignacien à l'Ahrensbourgien. Actes du colloque de Chaumont (17-18 octobre 1994). Reims, Société Archéologique Champenoise, p. 15-35 (Mémoire de la Société Archéologique Champenoise, $n^{\circ} 13$, suppl. 2). 
BROU L. 2009 - De l'Aurignacien au Trou de la Mère Clochette à Rochefort-sur-Nenon. In : L. Jaccottey, L. Brou (Eds.), Les recherches de Julien Feuvrier (1851-1936), historien, archéologue, archiviste et conservateur du musée : Catalogue d'exposition «Dole et sa région, de la Préhistoire au 18e siècle ". Musée des Beaux-Arts de Dole, 13 mars-24 mai 2009, sous la direction de Jean-Luc Mordefroid, Jaccottey L. \& Brou L. (Commissariat scientifique). Dole, Association des Amis du musée du Jura, p. 65-71.

CHIOTTI L. 2003 - Les productions lamellaires dans l'Aurignacien de l'abri Pataud, Les Eyzies-de-Tayac (Dordogne), Gallia préhistoire, 45, p. 113-156.

COMBIER J. 1955 - Observations complémentaires sur le Périgordien. Bulletin de la Société préhistorique française, 52, 9, p. 602-603.

COMBIER J. 1956 - Solutré. Les fouilles 1907 à 1925. Travaux du Laboratoire de Géologie de la faculté des Sciences de Lyon, nouvelle série 2. Lyon.

COMBIER J. 1959 - Mellecey. Informations archéologiques. Gallia Préhistoire, 2, 120-121, p. 124.

COMBIER J. 1989 - Aurignacien et Périgordien dans l'Est de la France. In : J.-P. Mohen (Ed.), Le temps de la Préhistoire I. Paris : Faton, p. 274-275.

COMBIER J. 1990 - De la fin du Moustérien au Paléolithique supérieur- les données de la région Rhodanienne. In : C. Farizy (Ed.), Paléolithique moyen récent et Paléolithique supérieur ancien en Europe. Colloque International de Nemours (9-11 mai 1988), p. 267277 (Mémoires du Musée de Préhistoire d'lle-de-France, $\left.\mathrm{n}^{\circ} 3\right)$.

CONNET N. 2002 - Le Châtelperronien. Réflexions sur l'unité et l'identité techno-économique de l'industrie lithique. L'apport de l'analyse diachronique des industries lithiques des couches Châtelperroniennes de la grotte du Renne à Arcy-sur-Cure (Yonne). Lille : Université de Lille I, 2002. Thèse de doctorat, $445 \mathrm{p}$.

DELOZE V., DEPAEPE P., GOUÉDO, J.-M., KRIER V., LOCHT J. 1994 - Le Paléolithique dans le nord du Senonais (Yonne). Contexte géomorphologique, industries lithiques et chronostratigraphie. documents d'Archaeologie Française, Vol. 47. Maison des sciences de l'Homme, Paris.

DELPORTE H. 1953 - L'industrie de Châtelperron et son extension géographique. In : Congrès Préhistorique de France. Compte-rendu, XIVème session, Strasbourg-Metz, p. 233-249.

DELPORTE H. 1955 - De la complexité du fait paléolithique à la lumière de fouilles nouvelles (Châtelperron et Germolles). Pallas, 3, Faculté des lettres, Toulouse, p. 153-162.
DEMARS P.-Y., LAURENT P. 1989 - Types d'outils lithiques du paléolithique supérieur en Europe. Paris: Centre National de la Recherche Scientifique (Cahiers du Quaternaire, $n^{\circ} 14$ ).

DESBROSSE R. 1976 - Le Paléolithique supérieur dans le Jura et en Franche-Comté. La Préhistoire Française I, 2, Paris : CNRS Éditions, p. 1348-1357.

DESBROSSE R. 1982 - Sites périgordiens en grottes dans le quart nord-est de la France. In: Aurignacien et Gravettien en Europe. Liège : Université de Liège, p. 105-122 (Etudes et Recherches Archéologiques de l'Université de Liège, $n^{\circ}$ 13: 2).

DESBROSSE R. 1984 - Périgordien et Aurignacien anciens de la Mère Clochette à Rochefort-sur-Nenon (Jura). In : Éléments de préhistoire et protohistoire européenne. Hommages à Jacques-Pierre Millotte. Paris : Belles Lettres. Annales littéraires de l'université de Besançon, p. 71-95 (Série archéologie $n^{\circ} 32$ ).

DESBROSSE R., TEXIER P. J. 1973 - Les silex de Germolles dans la collection Jeannin. La Physiophile, 79, p. 64-70.

DESBROSSE R., KOZLOWSKI J. K., ZUATE Y ZUBER, J. 1976 - Prondniks de France et d'Europe Centrale. L'Anthropologie, 80, 3, p. 431-448.

DUTKIEWICZ E. 2011 - Die Grotte de La Verpillière I - 150 Jahre Forschungsgeschichte. Die Aufarbeitung und Auswertung der Altgrabungen des paläolithischen Fundplatzes Germolles (Commune de Mellecey, Saône-etLoire, Frankreich). Tübingen: Eberhard Karls Universität Tübingen, 2011. Mémoire de Maîtrise, 179 p.

DUTKIEWICZ E., FLOSS H. 2015 - La grotte de La Verpillière I à Germolles, site de référence paléolithique en Bourgogne méridionale. Historique des 150 ans de recherches. La Physiophile, 162, p. 13-32.

FISCHER A., VEMMING HANSEN P., RASMUSSEN P. 1984 - Macro and Micro Wear Traces on Lithic Projectile Points. Experimental Results and Prehistoric Examples. Journal of Danish Archaeology, 3, p. 19-46.

FLOSS H. 2003 - Did they meet or not? Observations on Châtelperronian and Aurignacian Settlement Patterns in Eastern France. In: J. Zilhao, D'Errico F. (Ed.), The Chronology of the Aurignacian and of the Transitional Technocomplexes : Dating, Stratigraphies, Cultural Implications. Actes du symposium 6.1 du Congress XIV de I'UISPP (2-8 Septembre 2001), Lisbonne: Instituto Português de Arqueologia, p. 273-287, (Trabalhos de arqueologia, $n^{\circ} 33$ ).

FLOSS H. 2005 - Das Ende nach dem Höhepunkt. Überlegungen zum Verhältnis Neandertaler - anatomisch moderner Mensch auf Basis neuer Ergebnisse zum Paläolithikum in Burgund. In: N. J. Conard, S. Kölbl, W. Schürle (Eds.), Vom Neandertaler zum modernen Menschen, Ostfildern : Thorbecke, p. 109-130. 
FLOSS H. et al. 2006 à 2016 - Rapports de fouilles programmées des grottes de La Verpillière à Germolles (commune de Mellecey, Saône-et-Loire), DRAC, S.R.A. Bourgogne, Dijon.

FLOSS H., DUTKIEWICZ E., FRICK J.A., HOYER C.T. 2013 - Le Paléolithique supérieur ancien en Bourgogne du sud. In : P. Bodu, L. Chehmana, L. Klaric, L. Mevel, S. Soriano, N. Teyssandier (Eds.), Le Paléolithique supérieur ancien de l'Europe du Nord-Ouest: Réflexions et synthèses à partir d'un projet collectif de recherche sur le centre et le sud du Bassin parisien. Actes du colloque de Sens (15-18 avril 2009), Paris : Société Préhistorique Française, p. 331-350.

FLOSS H., HOYER C.T., HECKEL C.E., TARTAR É. 2015 a - The Aurignacian in Southern Burgundy. P@léthnologie, 7, p. 165-187.

FLOSS H., HOYER C.T., PAUTRAT J.-Y. 2015 b - De Néanderthal à Cro-Magnon. Un PCR, consacré au Paléolithique en Bourgogne Méridionale, In: S.R.A Bourgogne, ARTeHIS (Eds.), Actes de la Journée Regionale d'Archéologie. (24 mai 2014). Dijon, Imprimerie Chaumeil, p. 44-50.

FRICK J.A., FLOSS H. 2017 - Analysis of bifacial elements from Grottes de La Verpillière I \& II (Germolles, France). Quaternary International, 428, p. 3-25. doi:10.1016/ j.quaint.2015.10.090.

GÖTZ T. 2013 - Das Jungpaläolithikum der Grotte de La Verpillière II (Burgund, Frankreich). Tübingen : Eberhard Karls Universität Tübingen, 2013. Thèse de bachelor, 55 p.

GROS A.-C. 1958 - La Grotte de La Verpillière à Germolles (Saône-et-Loire). L'Eduen, 8, p. 7-8.

GROS A.-C. 2007 - Henri Delporte et les fouilles de la grotte de Germolles (Saône-et-Loire). In : R. Desbrosse, A. Thévenin (Eds.), Arts et cultures de la préhistoire, Paris : CTHS, p. 159-167 (Documents préhistoriques, $n^{\circ} 24$ ).

GROS O., GROS A.-C. 2005 - Le Chalonnais préhistorique. Collections du Musée de Chalon-sur-Saône. Chalon-sur-Saône. Chalon-sur-Saône.

GUILLARD E. 1954 - Une station aurignacienne inédite à Germolles. Mémoires de la Société d'Histoire et d'Archéologie de Chalon-sur-Saône, 33, p. 129-138.

GUILLARD E. 1959 - Note sur les stations et vestiges préhistoriques de la Côte Chalonnaise trouvés à Chenoves, Saules et à l'est de Culles-les-Roches. La Physiophile, 50, p. 2-16.

HAHN J. 1993 - Erkennen und Bestimmen von Stein- und Knochenartefakten. Einführung in die Artefaktmorphologie. Tübingen : Institut für Urgeschichte (Archaeologica Venatoria, $\mathrm{N}^{\circ} 10$ ).
HARROLD F. B. 1986 - Une réévaluation du Châtelperronien. Bulletin de la Société Préhistorique Ariège-Pyrnénées, 41, p. 151-169.

HECKEL C., HIGHAM T.F.G., HOYER C.T., FLOSS H. 2016 - Radiocarbon dating of Verpillière I and II. In: Project Collectif de Recherche: Le Paléolithique supérieur ancien en Bourgogne méridionale. Genèse, chronologie et structuration interne, évolution culturelle et technique. Raport anuell 2015. Abteilung für Ältere Urgeschichte und Quartärökologie, Service Régional d'Archéologie Bourgogne, Tübingen, Dijon, p. 30-37.

HERKERT K., SIEGERIS M., CHANG J.-Y., CONARD N. J., FLOSS H. 2015 - Zur Ressourcennutzung später Neandertaler und früher moderner Menschen. Fallbeispiele aus dem südlichen Burgund und der Schwäbischen Alb. Mitteilungen der Gesellschaft für Urgeschichte, 24, p. 141-172.

HIGHAM T., BASELL L., JACOBI R., WOOD R., BRONK RAMSEY C., CONARD N. J. 2012 - Testing models for the beginnings of the Aurignacian and the advent of figurative art and music: The radiocarbon chronology of Geißenklosterle, Journal of Human Evolution, 62, p. 664--676.

HOYER C.T., HUBER N., LITZENBERG R., FLOSS H. 2016 - Rapport de Fouille 2013-2015. In: Fouilles programmés pluriannuelles aux sites paléolithiques des Grottes de La Verpillière I \& II à Germolles, commune de Mellecey (Saône-et-Loire). Rapport annuel 2015, rapport pluriannuel 2013 à 2015. Abteilung für Ältere Urgeschichte und Quartärökologie, Service Régional d'Archéologie Bourgogne, Tübingen, Dijon, p. 86-133.

HUBLIN J.-J., TALAMO S., JULIEN M., DAVID F. CONNET N., BODU P., VANDERMEERSCH B., RICHARDS M.P. 2012 - Radiocarbon dates from the Grotte du Renne and Saint-Césaire support a Neandertal origin for the Châtelperronian, PNAS, 109 : 46, p. 1874318748.

JOLY J 1959 - St. Aubin. Gallia Préhistoire, 2, p. 96-98.

KREMMER A. 2015 - Die Lamellen aus dem GH1 der paläolithischen Fundstelle Grotte de La Verpillière I bei Germolles, Burgund. Eine statistische und typologische Auswertung. Tübingen : Eberhard Karls Universität Tübingen, 2015. Thèse de bachelor, $92 \mathrm{p}$.

LITZENBERG R. 2015 - Materialübergreifende Analyse des GH 16 der Grotte de La Verpillière I in Germolles, Gemeinde Mellecey (Saône-et-Loire, Frankreich). Tübingen : Eberhard Karls Universität Tübingen, 2015. Thèse de bachelor, $97 \mathrm{p}$.

MÉRAY CH. 1869 - L'âge de la Pierre à Germolles Matériaux d'archéologie et d'histoire. Chalon sur Saône, p. 83-86.

MÉRAY CH., CHABAS F. 1876 - Compte rendu des fouilles de la caverne de Germolles et notes additionnelles. 
Mémoires de la société d'histoire et d'archéologie de Chalon-sur-Saône, 6, Ile partie, p. 251-266.

O'FARRELL M. 2004 - Les pointes de la Gravette de Corbiac (Dordogne) et considérations sur la chasse au Paléolithique supérieur ancien. In : Approches fonctionnelles en Préhistoire. Actes du XXV Congrès Préhistorique de France, Nanterre, 24-26 novembre 2000, p. 121-138.

O'FARRELL M. 2005 - Étude préliminaire des éléments d'armature lithique de l'aurignacien ancien de Brassempouy. In: F. Le Brun-Ricalens, J.-G. Bordes, F. Bon (Eds.), Productions lamellaires attribuées à l'Aurignacien : Chaînes opératoires et perspectives technoculturelles. XIVe congrès de I'UISPP, Liège (2-8 Septembre 2001). Luxembourg, Musée national d'Histoire et d'Art, p. 395-412 (ArchéoLogiques, $n^{\circ} 1$ ).

PELEGRIN J. 1995 - Technologie Lithique. Le Châtelperronien de Roc-De-Combe (Lot) et de La Côte (Dordogne). Paris: CNRS Éditions (Cahiers du Quaternaire, $\left.n^{\circ} 20\right)$.

PELEGRIN J. 2000 - Les techniques de débitage laminaire au Tardiglaciaire : critères de diagnose et quelques réflexions. In : B. Valentin, $\mathrm{P}$. Bodu et $\mathrm{M}$. Christensen (Eds.), L'Europe centrale et septentrionale au Tardiglaciaire. Confrontation des modèles régionaux de peuplement. Actes de la table-ronde de Nemours, (mai 1997). Nemours, APRAIF, Mémoire du Musée de Préhistoire d'lle-de-France, 7 , p. 73-86

PELEGRIN J. 2012 - Sur les débitages laminaires du Paléolithique supérieur. In : F. Delpech et J. Jaubert (Eds.), François Bordes et la Préhistoire. Colloque international François Bordes, Bordeaux (22-24 avril 2009). Paris, Édit. du CTHS, p. 141-152. (Documents préhistoriques, 29) ISBN 978-2-7355-0766-5.

PELEGRIN J. et SORESSI M. 2007 - Le Châtelperronien et ses rapports avec le Moustérien. In : B. Vandermeersch et B. Maureille (dirs). Les Néandertaliens. Biologie et cultures. Paris : Editions du CTHS, p. 283-296 (coll. Documents préhistoriques, 23).

PESESSE D., MICHEL A. 2006 - Le burin des Vachons : apports d'une relecture technologique à la compréhension de l'Aurignacien récent du nord de l'Aquitaine et des Charentes. PalEo, 18, p. 143-160.

RICHARD M., FALGUÈRES C., GHALEB B., RICHTER D. 2016 a - Résultats préliminaires des datations par ESR/UTh sur émail dentaire, Grotte de La Verpillière I \& II, In: Project Collectif de Recherche: Le Paléolithique supérieur ancien en Bourgogne méridionale. Genèse, chronologie et structuration interne, évolution culturelle et technique. Rapport annuelle 2015. Abteilung für Ältere Urgeschichte und Quartärökologie, Sérvice Régional d'Archéologie Bourgogne, Tübingen, Dijon, p. 11-23.

RICHARD M., FALGUÈRES C., GHALEB D., ZÖLLER L., FRICK J.A., HOYER C., FLOSS H. 2016 b - Enjeux et méthodologie des datations par ESR/U-Th de sites de la fin du Paléolithique Moyen. Présentation des données préliminaires des Grottes de La Verpillière I et II. Poster Q10 AFEQ CNF-INQUA, Paléoclimats et environnements quaternaires, quoi de neuf sous le soleil ? Bordeaux (16-18 février 2016).

RIOS-GARAIZAR J. 2008 - Nivel IX (Chatelperroniense) de Labeko Koba (Arrasate-Gipuzkoa): gestión de la industria lítica y función del sitio, Munibe, 59, p. 25-46.

RIOS-GARAIZAR J., LIBANO SILVENTE I., GARATE MAIDAGAN D. 2012 - El yacimiento chatelperroniense al aire libre de Aranbaltza (Barrika, Euskadi). Munibe, 63, p. 81-92.

ROUSSEL M. 2011 - Normes et variations de la production lithique Durant le Châtelperronien: la séquence de la Grande-Roche-de-la-Plématrie à Quinçay (Vienne). Thèse de doctorat en Préhistoire de l'Université Paris OuestNanterre, 2011. 540p.

ROUSSEL M. 2013 - Méthodes et rythmes du débitage laminaire au Châtelperronien: comparaison avec le ProtoAurignacien. Comptes Rendus Palevol, 12, p. 233-241.

ROUSSEL M. 2014 - Des Lamelles retouchées au Châtelperronien : Diffusion d'Idées entre derniers Néandertaliens et premiers Hommes modernes migrants. In : M. Otte, F. Le Brun-Ricalens (Eds.), Modes de contacts et de déplacements au paléolithique eurasiatique. Actes du Colloque international de la commission 8 (Paléolithique supérieur) de I'UISPP (28-31 mai 2012), Liège, Université de Liège, p. 491-510.

ROUSSEL M., SORESSI M. 2010 - La Grande Roche de la Plématrie à Quinçay (Vienne). L'évolution du Châtelperronien revisitée. In : J. Buisson-Catil, J. Primault (Eds.), Préhistoire entre Vienne et Charente. Hommes et sociétés du Paléolithiques. Chauvigny, Association des Publications Chauvinoises, p. 203-219.

ROUSSEL M., SORESSI M. 2014 - Le Châtelperronien. In: Otte, M.(Ed.), Néandertal - Cro Magnon. La Rencontre. Arles, Editions Errance, p. 31-59.

ROUSSEL M., SORESSI M., HUBLIN J.-J. 2016 - The Châtelperronian conundrum: Blade and bladelet lithic technologies from Quinçay, France. Journal of Human Evolution, 95, p. 13-32.

RUEBENS K., MCPHERRON S.J.P., HUBLIN J.-J. 2015 On the local Mousterian origin of the Châtelperronian: Integrating typo-technological, chronostratigraphic and contextual data. Journal of Human Evolution, 86, p. 55-91.

SZMIDT C., BROU L., JACCOTTEY L. 2010 - Direct radiocarbon (AMS) dating of split-based points from the (Proto)Aurignacian of Trou de la Mère Clochette, Northeastern France. Implications for the characterization of the Aurignacian and the timing of technical innovations in Europe. Journal of Archaeological Science 37 (12): 3320-3337. 
H. FLOSS, Ch. HOYER, H. WÜRSCHEM

SORESSI M. 2002 - Le Moustérien de tradition acheuléenne du sud-ouest de la France. Discussion sur la signification du faciès à partir de l'étude comparée de quatre sites : Pech-de-l'Azé I, Le Moustier, La Rochette et la Grotte XVI. . Thèse de doctorat en Géologie-Préhistoire de l'Université de Bordeaux I, 2002. 330 p.

SORESSI M., ROUSSEL M. 2014 - European Middle to Upper Paleolithic Transitional Industries : Châtelperronian. In: C. Smith (Ed.), Encyclopedia of Global Archaeology. New York, Springer, p. 2679-2693.

SONNEVILLE-BORDES D. (de), PERROT J. 1956 Lexique typologique du Paléolithique supérieur. Bulletin de la Société préhistorique française, 53, 9, p. 547-559.

TARTAR E., HECKEL C. 2016 - Les productions en matières osseuses de la grotte de La Verpillière I. In: Fouilles programmés pluriannuelles aux sites paléolithiques des Grottes de La Verpillière I \& II à Germolles, commune de Mellecey (Saône-et-Loire). Rapport annuel 2015, rapport pluriannuel 2013 à 2015. Abteilung für Ältere Urgeschichte und Quartärökologie, Service Régional d'Archéologie Bourgogne, Tübingen, Dijon, p. 191-217.

VILLA P., BOSCATO P., RANALDO F., RONCHITELLI A. 2009 - Stone tools for the hunt: points with impact scars from a Middle Paleolithic site in southern Italy. Journal of Archaeological Science, 36, p. 850-859.
WEGENG H. 2015 - Das Protoaurignacien von Germolles (Saône-et-Loire, Frankreich). Tübingen : Eberhard Karls Universität Tübingen, 2015. Thèse de bachelor, $100 \mathrm{p}$.

WEGENG H., FLOSS H. 2016 - The Proto-Aurignacian in the Grotte de La Verpillière I (Germolles, Saône-et-Loire). In : Rapport annuel 2015, rapport pluriannuel 2013 à 2015. Abteilung für Ältere Urgeschichte und Quartärökologie, Service Régional d'Archéologie Bourgogne, Tübingen, Dijon, p. 175-187.

WÜRSCHEM H. 2015 - Das Châtelperronien der Grotte de La Verpillière I (Saône-et-Loire, Frankreich). Tübingen : Eberhard Karls Universität Tübingen, 2015. Thèse de bachelor, $133 \mathrm{p}$.

ZÖLLER L., SCHMIDT C. 2016 - Germolles - Grotte de La Verpillière II - GH 3 \& 4, Report on luminescence dating of cave sediments. In: Project Collectif de Recherche: Le Paléolithique supérieur ancien en Bourgogne méridionale. Genèse, chronologie et structuration interne, évolution culturelle et technique. Rapport annuel 2015. Abteilung für Ältere Urgeschichte und Quartärökologie, Service Régional d'Archéologie Bourgogne, Tübingen, Dijon, p. 45-49. 